\title{
Blue Phase III: Topological Fluid of Skyrmions
}

\author{
J. Pišljar@, ${ }^{1}$ S. Ghosh, ${ }^{2}$ S. Turlapati, ${ }^{3}$ N. V. S. Rao, ${ }_{5}^{3}$ M. Škarabot, ${ }^{1}$ A. Mertelj $\odot,{ }^{4}$ A. Petelin,,${ }^{5,4}$ A. Nych®,${ }^{1,6}$ \\ M. Marinčič $\odot,{ }^{5,1}$ A. Pusovnik, ${ }^{5}$ M. Ravnik ${ }^{5,1}$ and I. Muševič $\circledast^{1,5, *}$ \\ ${ }^{1}$ Condensed Matter Department, J. Stefan Institute, Jamova 39, SI-1000 Ljubljana, Slovenia \\ ${ }^{2}$ Department of Physics, University of Calcutta, \\ 92 Acharya Prafulla Chandra Road, Kolkata 700 009, India \\ ${ }^{3}$ Chemistry Department, Assam University, Silchar 788011, Assam, India \\ ${ }^{4}$ Department of Complex Matter, J. Stefan Institute, Jamova 39, SI-1000 Ljubljana, Slovenia \\ ${ }^{5}$ Faculty of Mathematics and Physics, University of Ljubljana, Jadranska 19, SI-1000 Ljubljana, Slovenia \\ ${ }^{6}$ Department of Molecular Photoelectronics, Institute of Physics, Nauky prospect 46, Kyiv, 03680, Ukraine
}

(Received 26 July 2021; revised 12 October 2021; accepted 12 November 2021; published 5 January 2022)

\begin{abstract}
Skyrmions are topologically protected, vortexlike formations of a field that cannot be removed by any smooth transformation and emerge in a range of fundamentally different, either quantum or classical systems, from spin textures to chiral ferromagnets and chiral complex fluids. Notably, they are generally observed in thin ordered or disordered quasi-2D layers, but little is known about their three-dimensional structuring and organization, including structural transitions from 2D to 3D. Here, we show experimentally and numerically that the blue phase (BP) III of a chiral liquid crystal is a 3D fluid of chiral skyrmion filaments of the nematic orientational field, entangled with a 3D network of topological defect lines. It is an effective 3D dynamic fluid determined by the thermal fluctuations of two distinct branches of excitations: rapid internal fluctuations of the skyrmion structure and a slow collective motion of the skyrmion filaments. When confined to less than an approximately $150-\mathrm{nm}$ layer, the 3D bulk skyrmion fluid transforms into a different effectively 2D liquid of half-skyrmions, with the dynamics of the skyrmion liquid slowing down by an order of magnitude and with the individual skyrmions lingering, and even disappearing into, and reappearing from the homogeneous liquid crystal. The thickness-temperature phase diagram actually shows that both the BPIII and BPI phases are made of skyrmions, which when confined to less than approximately $150 \mathrm{~nm}$ cells transform equally into a 2D half-skyrmion liquid. The temperature range of this $2 \mathrm{D}$ half-skyrmion liquid is much broader than the temperature interval of BP phases, which makes BP materials interesting for broad-temperature-range skyrmionic applications. We envisage a soft matter skyrmionic device, in which skyrmions are created and detected by light.
\end{abstract}

DOI: $10.1103 /$ PhysRevX.12.011003

Subject Areas: Soft Matter

\section{INTRODUCTION}

Originally proposed to describe the stability of the constituents of elementary particles [1], skyrmions are vortexlike formations of a field appearing in a number of different systems [2]. They can be observed as particlelike configurations of the velocity field in a superfluid $\mathrm{He}^{3}-A$ phase [3], as 2D spin textures in the quantum Hall effect [4] and spinor Bose-Einstein [5] condensates (BECs). In solid state, quasi-two-dimensional skyrmions are vortexlike configurations of the magnetization vector

\footnotetext{
* Corresponding author. igor.musevic@ijs.si

Published by the American Physical Society under the terms of the Creative Commons Attribution 4.0 International license. Further distribution of this work must maintain attribution to the author(s) and the published article's title, journal citation, and DOI.
}

field in chiral ferromagnets [6,7] or magnetic whirls with a ferroelectric polarization [8]. Skyrmions are topologically protected structures, which means that they cannot be transformed into another texture without breaking or melting the field. Topological protection, therefore, implies an energy barrier, which needs to be overcome to remove the skyrmions, and this makes them interesting for application in information storage and processing. Because of their small, nanometric size, compactness, and extremely high resonant frequencies, magnetic skyrmions could provide new route to high-density memory devices.

Skyrmions are abundant in chiral liquid crystals (CLCs), where the chirality of the constituent molecules is responsible for spontaneous solitonic deformations of the tensorial orientational field of chiral nematic liquid crystals [9-11]. The symmetry of the liquid medium allows for a free-energy contribution that is proportional to $\boldsymbol{n} \cdot(\nabla \times \boldsymbol{n})$, where $\boldsymbol{n}$ is the director field describing local orientation of LC molecules. This term is identical to the 


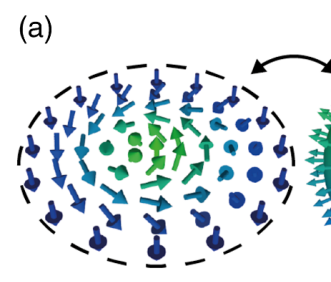

(d)

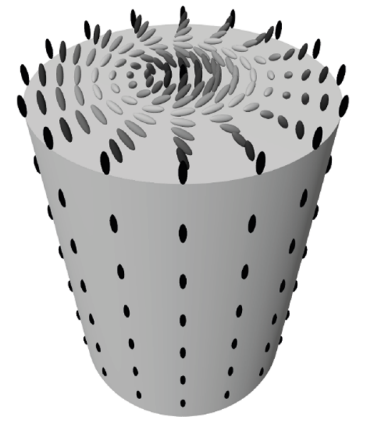

(b)

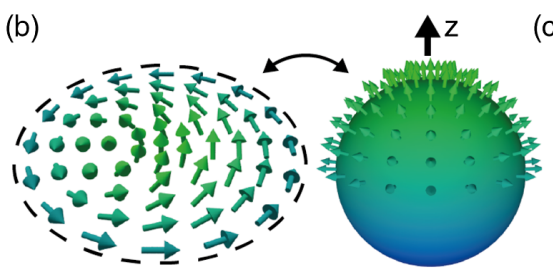

(e)

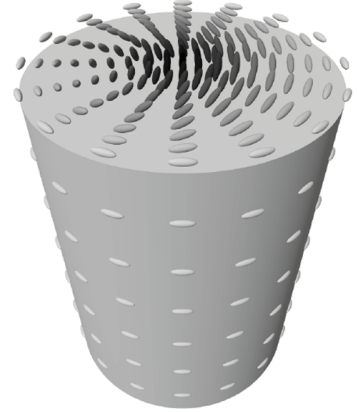

(c)

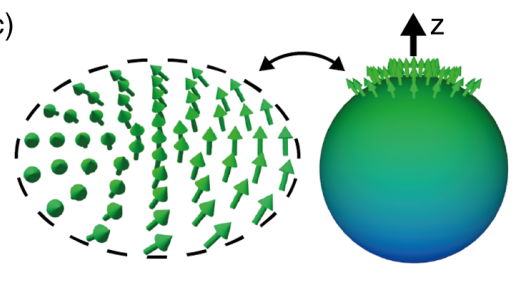

(f)

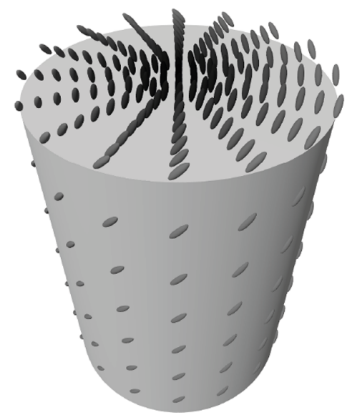

FIG. 1. Full, half-, and quarter-skyrmions in 2D and skyrmion filaments in 3D. (a) 2D vector field structure of a full skyrmion with topological charge $q=1$. The sphere on the right-hand side shows the corresponding vector field mapping on a sphere. The vector field of a full skyrmion (left) assumes every possible orientation in 3D only once, as shown on the sphere. (b) 2D vector field of a halfskyrmion, also named meron, with fractional topological charge of $q=1 / 2$. The vector field of such a skyrmion assumes one-half of possible orientations in 3D space, as shown on the right-hand sphere. (c) 2D vector field of a quarter-skyrmion with fractional topological charge of $q=1 / 4$. (d)-(f) Director field structures $\boldsymbol{n}(\boldsymbol{r})$ of full-, half-, and quarter-skyrmion filaments, respectively. The local orientation of LC molecules is represented using ellipsoids shaded according to the spatially varying $z$ component, where darker shades denote higher values of the $z$ component (i.e., along the skyrmion filament). The straight filament shown in (f) is also termed the double-twist cylinder.

Dzyaloshinsky-Moriya [12,13] interaction term in ferromagnets and Rashba spin-orbit coupling [14] in BECs. It induces spontaneous twisting of the nematic order parameter field, which self-shapes into various skyrmionic structures. Skyrmions are indeed observed in nematic CLCs, either as stable and isolated topological objects [15], as skyrmionic bags [16], or as assemblies of hundreds of skyrmions [17]. When skyrmions are elongated into filaments, the double-twist cylinders (DTCs) are formed, which are proposed to form thermodynamically stable blue phases I and II (BPI and BPII) [18] of highly chiral LC materials.

Skyrmions are usually characterized by their integer topological charge $q$ (also called winding or skyrmion number), describing the number of times the skyrmion vector field wraps the unit sphere of all possible orientations in 3D space [19]. An elementary 2D skyrmion, also called full skyrmion, has the topological charge $q=1$ and is shown in Fig. 1(a). In a full skyrmion, the director in a LC (or magnetization vector in magnets) rotates for an angle $\pi$ as we move from the skyrmion center radially outward. Full skyrmions can be smoothly embedded in 2D geometry, and no defects are needed to compensate for the winding inside skyrmions [5,6]. In liquid crystals, stable full skyrmions are theoretically treated by Bogdanov, Rößler, and Shestakov [10]. Two-dimensional skyrmions (also called "baby skyrmions") and other solitonic structures are observed in confinement-frustrated chiral nematics by Ackerman et al. [15].
A 2D half-skyrmion is characterized by a fractional number $|q|=1 / 2$, and the radial twist is $\pi / 2$, as shown in Fig. 1(b). Half-skyrmions, also named merons [20], cannot be packed in 2D without additional singularities, and defects such as $-1 / 2$ winding number defect lines are formed in regions where the fields from neighboring skyrmions meet and the field is not able to make a smooth transition in space. Thermodynamically stable halfskyrmions are observed in magnetic materials, where they form single objects or 2D lattices $[21,22]$. In chiral LCs, half-skyrmions are observed in thin layers of BPI LCs, where they not only form a hexagonal 2D crystal, but also appear as isolated objects [23].

While full and half-skyrmions are observed in thin layers of liquid crystals, forming an effectively 2D system, little is known about the stability of other types of fractional skyrmions, such as quarter-skyrmions, or even real-number skyrmions, in 2D and 3D. Quarter-skyrmion filaments with fractional topological charge $|q|=1 / 4$ [Figs. 1(c) and 1(f)] are filaments with a cross section that shows a $\pi / 4$ radial twist. Alternatively, they are often named DTCs. They are not stable in 3D but could be stable in 3D cylindrical confinement. DTCs are proposed by Saupe [24] to form three-dimensional crystals of blue phases I and II. In BPI and BPII, the quarter-skyrmion filaments are considered to be arranged in body-centered and simple cubic lattices, respectively, which are intertwined with a lattice of topological defect lines with a $-1 / 2$ winding. The skyrmion structure of BPI was recently confirmed by confining the 
BPI material into very thin layers, where the bulk BPI skyrmion filaments transform into half-skyrmions forming a hexagonal lattice [23].

The third member of the BP family, also termed the BPIII phase, was first reported by Stegemeyer and Bergman [25] and later by Marcus [26]. It is proposed by Marcus that BPIII is an isotropic melt of DTCs, and this is supported by the calculations of optical rotatory power by Collings on a model made of randomly oriented small BPII domains [27]. BPIII is a bluish, seemingly featureless, optically isotropic liquid that is identified as a thermodynamically stable liquid phase in a number of compounds by calorimetric [28-30], light-scattering [30-33], and optical measurements [33-35]. It exists in a usually narrow window of the chirality-temperature phase diagram [36,37], with a BPIII-isotropic critical point similar to the vapor liquid critical point identified from calorimetric and optical data $[28,38]$. Icosahedral or bond orientational models of the BPIII phase structure are suggested by various theories [39-42] but not confirmed experimentally. Mesoscopic numerical simulations $[43,44]$ predict the structure of BPIII as an amorphous network of disclination lines permeated by double-twisted regions, which is in agreement with the proposal by Marcus [26]. Although a range of evidence is consistent with BPIII as a liquid tangle of DTCs [45-47], the microscopic structure of BPIII remains unclear, due to the lack of convincing experimental evidence. The smallness of DTCs and the strong thermal fluctuations of BPIII make it impossible to observe the DTCs in bulk BPIII directly.

Here, we show that the BPIII of a chiral liquid crystal is a dynamic, 3D liquid phase of skyrmion filaments (i.e., double-twist filaments) of the nematic orientational field $\boldsymbol{n}(\boldsymbol{r})$, entangled with a 3D network of singular topological defect lines. The manuscript is organized as follows. In Sec. II, we present dynamic behavior of 3D skyrmion fluid from BPIII, giving evidence of slow and fast fluctuations in bulk BPIII, which is a clear signature of well-formed and 3D-organized skyrmions. Section III describes the experiments where individual skyrmions of BPIII material are isolated and can be clearly observed, as confined to a cell so thin that it cannot accommodate but one skyrmion layer. Section IV describes the experimentally measured dynamics in very thin layers of BPIII, which gives evidence of long-lived half-skyrmions with lifetimes of the order of 10 s. Landau-de Gennes modeling of skyrmion structures is described in Sec. V, which, together with optical simulation of their appearance under an optical microscope, is in full agreement with experiments, further confirming that BPIII is a 3D topological liquid of skyrmion filaments. Discussion on the open theoretical questions which emerge from our experiments is given in Sec. VI. Finally, we discuss in Sec. VII possible application of BP skyrmions, presenting the competing advantages of soft matter skyrmionics over the magnetic skyrmions in solid state.

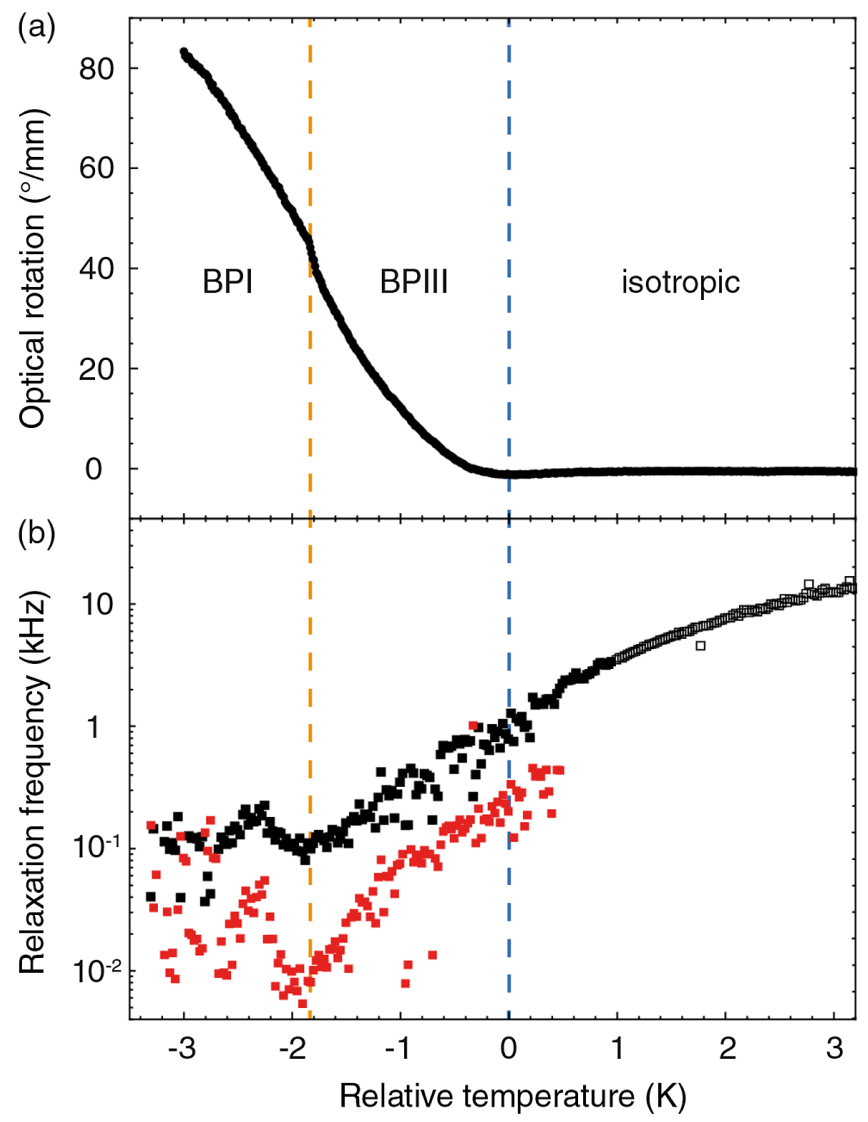

FIG. 2. Optical rotation and dynamics of BPIII phase. (a) Temperature dependence of the angle of polarization rotation for a linearly polarized light passing through a $140-\mu \mathrm{m}$-thick cell of the BPIII mixture. Strong optical activity clearly marks the isotropic BPIII transition and shows discontinuity at the BPIII-BPI transition. (b) Spectrum of order-parameter fluctuations across the isotropic-BPIII-BPI phase transition in 50- $\mu$ m-thick BPIII LC measured by DLS. The zero of the ORP and DLS temperature scales is set to the isotropic BPIII and is determined by calibrating ORP and DLS setups using isotropic-nematic transition of 5CB.

\section{DYNAMIC BEHAVIOR OF BULK 3D SKYRMION FLUID}

The experiments are performed in a BPIII LC mixture, which upon cooling exhibits a continuous transition from the isotropic phase to the BPIII at approximately $29^{\circ} \mathrm{C}$, a first-order phase transition into the BPI at approximately $27.9^{\circ} \mathrm{C}$, and another first-order transition to the cholesteric phase at approximately $25.5^{\circ} \mathrm{C}$ (see the Appendix A for details). The temperatures of these phase transitions are most conveniently determined from the measured temperature dependence of the optical rotatory power (ORP) in a 140- $\mu$ m-thick sample, as presented in Fig. 2(a) (for details see Appendix B). There is a clear and continuous onset of the ORP at the isotropic-BPIII phase transition, indicating the second-order, i.e., continuous, nature of this transition. In addition, there is no BPII phase between BPI and BPIII in our BPIII mixture, which indicates that the 
BPIII-isotropic phase transition is close to the critical point or in the supercritical region of chirality-temperature phase diagram $[30,32,35,38]$.

In the region of BPIII, very strong fluctuations can be observed in a thick LC sample under an optical microscope (see Supplemental Video 1 [48]). These fluctuations are so slow that they can easily be seen by the bare eye. The intensity of these fluctuations gradually increases throughout the isotropic-BPIII transition, in agreement with continuous onset of the ORP in Fig. 2(a).

To analyze the spectrum of fluctuations in the BPIII phase, we use the dynamic light scattering (DLS) technique and measure the photon autocorrelation function of the light, scattered from BPIII mixture, placed in a small rectangular glass capillary. We use a backscattering geometry with a relatively large scattering wave vector. The incoming light is right-handed circularly (RHC) polarized, and we detect only RHC-polarized scattered light as well. More details about the experimental setup, measuring protocols, and autocorrelation function analysis is presented in Appendix B.

Temperature dependence of the relaxation rates of the fluctuation modes visible in this RHC-RHC scattering geometry is shown in Fig. 2(b). In the isotropic phase, there is a single fluctuation mode, whereas, in the BPIII phase, another, slow mode is observed. The temperature at which this second slow mode appears coincides well with the isotropic-BPIII transition as observed in the ORP data; see Figs. 2(a) and 2(b). Both modes are slowing down when cooling through the BPIII phase, until the BPI is reached, where they remain practically constant with subsequent cooling (see Supplemental Video 6 [48] of the BPI-BPIII phase transition). The frequency of the slower mode is around $100 \mathrm{~Hz}$ at the isotropic-BPIII transition and lowers to tens of hertz in the BPIII, which makes these lowfrequency fluctuations visible with the bare eye. The amplitude of the slow mode is very small at the isotropic-BPIII transition and gradually increases by lowering the temperature. At the BPIII-BPI phase transition temperature, the slow and fast modes are of equal amplitude. Throughout the BPIII, there is a nearly one-order-ofmagnitude difference in the relaxation rates of the two modes, which makes the two-exponential fit of the measured autocorrelation functions of the scattered light intensity very reliable.

The two relaxation modes in BPIII are a rather striking observation. Although the importance of fluctuations for the stability of BPIII is stressed and taken into account in the theory [49], there is insufficient analysis of the spectrum of BPIII fluctuations in previous DLS studies [31,50-52]. The observation of two fluctuating modes in the BPIII phase is consistent with the picture of fluctuating liquid skyrmion filaments, as shown in Fig. 3: In the BPIII phase, the skyrmion filaments form a dynamic and strongly fluctuating liquid, which is completely disordered in terms

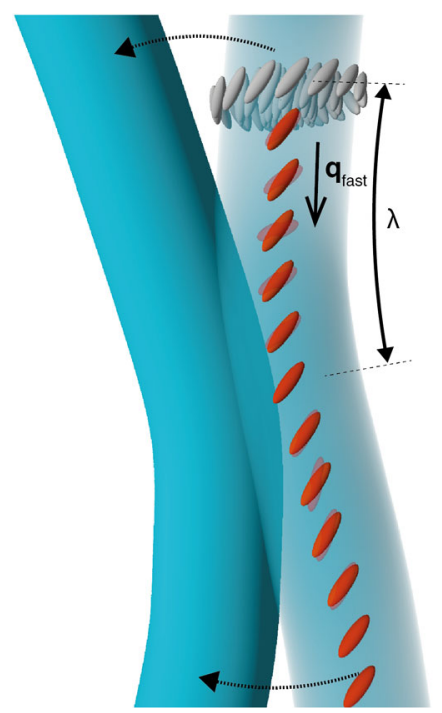

FIG. 3. Graphic illustration of fast and slow fluctuation modes in the BPIII phase. The slow mode represents positional and contour fluctuations of skyrmion filaments as a whole. The fast BPIII mode represents elastic deformation of the internal director structure of the filaments. This picture is to be considered as a conjecture only.

of shape and orientation. The slow mode corresponds to fluctuations of the position and shape of the skyrmion filaments, coupled to the disclination network, whereas the fast mode corresponds to twistlike structural fluctuations of the skyrmion filaments [32,33]. In such a case, the two relaxation modes should be observable in the thinnest layers of BPIII, where there is no place to accommodate more than a single layer of skyrmions.

In addition to ORP and DLS measurements on bulk BPIII samples, we measure the reflectivity of the BPIII in a broad temperature range to determine the lattice constant of BPI and the helical pitch of the cholesteric phase (details are in Appendix A). Figure 4(a) shows a photo of the bulk BPIII mixture taken in reflection from a glass capillary that is placed in a larger temperature gradient to observe all the phases of this BPIII mixture in the same photo. The BPIII phase appears as a deep-blue-colored, textureless liquid, which is well known from many experiments. There is no interface between the isotropic and BPIII phases in this photograph, indicating the continuous nature of the isotropic-BPIII transition. However, there is a clear interface between the BPIII and BPI phases, which is followed by a development of blue and green platelets in the lowertemperature region of BPI. The regular geometric shape of BPI platelets and their vivid color clearly indicate the crystalline nature of BPI, while the foggy blue appearance of BPIII indicates disordered, i.e., liquid, nature of BPIII.

We measure the temperature dependence of the spectrum of white light reflected from a thick sample (approximately $20 \mu \mathrm{m}$ ) of BPIII mixture sandwiched between two glass slides, which is shown in Fig. 4(b). The BPIII is found in a 

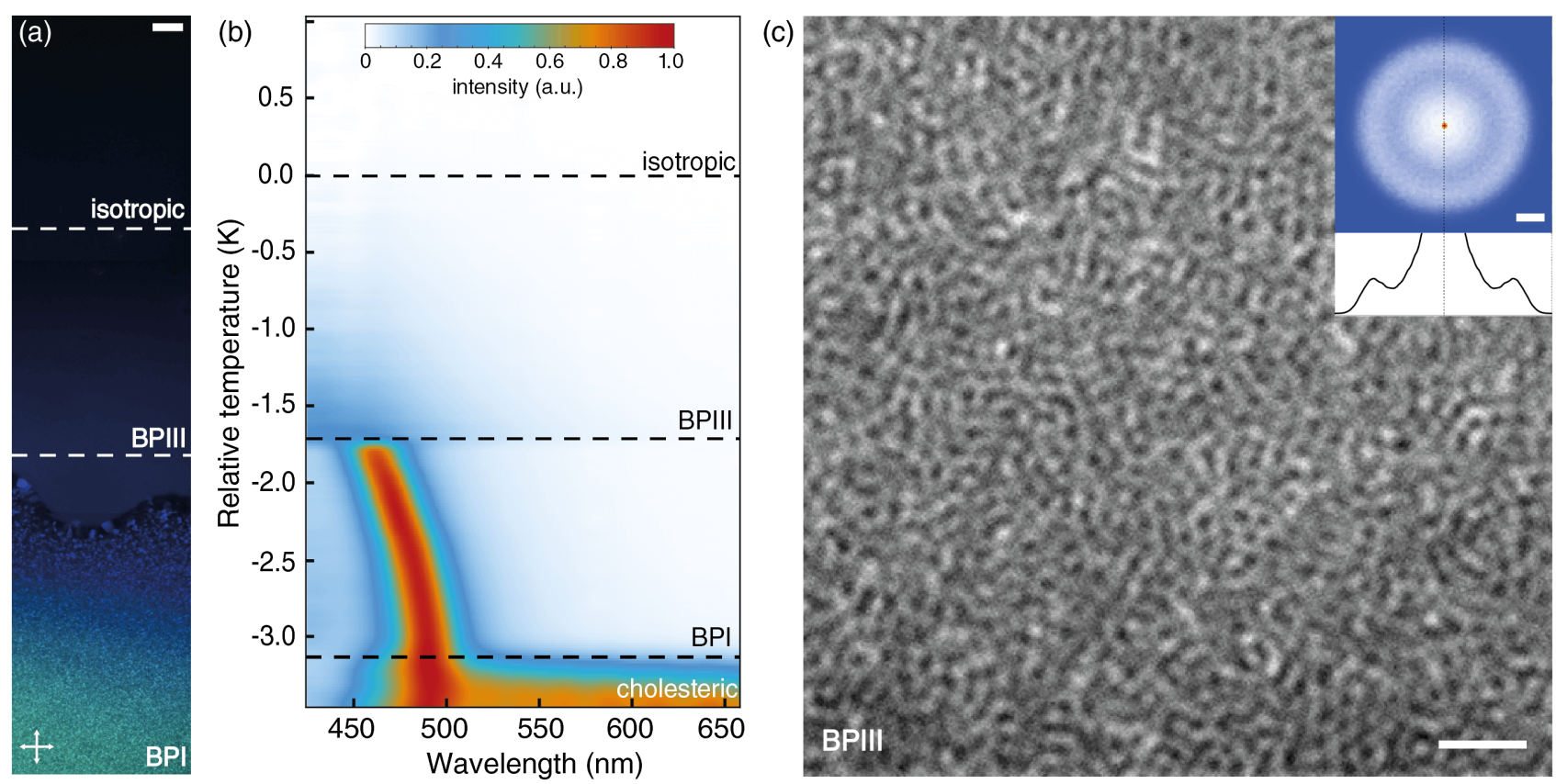

FIG. 4. Reflectivity and appearance of blue phase III of chiral liquid crystals. (a) Photograph of BPIII LC in a capillary with a large temperature gradient, from $T \sim 27^{\circ} \mathrm{C}$ at the bottom to $T \sim 30^{\circ} \mathrm{C}$ at the top. The scale bar indicates $10 \mu \mathrm{m}$. (b) Spectrum of white light reflected from a 20- $\mu$ m-thick layer of BPIII (see Appendix B). Note the continuous increase of the broad reflectivity spectrum of BPIII as it approaches the BPI. The Bragg reflection band in the BPI indicates long-range crystalline order with a lattice constant of approximately $215 \mathrm{~nm}$. (c) Photograph of BPIII taken in the reflection of green light from an approximately 1- $\mu$ m-thick cell. The dark spots are caused by the arrangement of skyrmion filaments near the surface. The scale bar indicates $1 \mu \mathrm{m}$. The inset is an average FFT of many BPIII images, showing an intensity ring centered at approximately $25 \mu \mathrm{m}^{-1}$, which corresponds to an approximately 250 -nm separation between the dark spots. The scale bar indicates $10 \mu \mathrm{m}^{-1}$.

narrow temperature region (approximately $1.8 \mathrm{~K}$ ) between the isotropic and BPI phases and is characterized by a faint but clearly detectable broad reflection band below approximately $550 \mathrm{~nm}$. In the crystalline BPI phase, the crystallike Bragg reflection peak is observed at a wavelength of approximately $470 \mathrm{~nm}$ at the BPIII $\rightarrow$ BPI transition, which is gradually redshifted to approximately $490 \mathrm{~nm}$ at the BPI to cholesteric transition.

A microphotograph of the BPIII phase taken at a short exposure time in an approximately $1-\mu \mathrm{m}$-thin cell is shown in Fig. 4(c). Although the BPIII is a strongly fluctuating phase, the short exposure time allows one to clearly see an amorphous, grainy structure of dark dots. After a Fourier transform of the image, a 2D structure factor in the form of a ring is revealed, as shown in the inset in Fig. 4(c). The maximum of the observable white ring in the inset in Fig. 4(c) is at approximately $25 \mu \mathrm{m}^{-1}$, which corresponds to an average distance of approximately $250 \mathrm{~nm}$ between the dark dots in Fig. 4(c). This average distance is well above the diffraction limit of our microscope, which is at approximately $190 \mathrm{~nm}$ and denotes the minimum detectable size of the objects on microscope images. The diffraction limit of the microscope is determined from the outer edge of the ring at approximately $33 \mu \mathrm{m}^{-1}$ in the inset in Fig. 4(c).
When the BPIII sample is observed in the reflection mode, the local optical contrast originates from the variation of the director in the focal plane of the microscope, and the optical contrast is strongest when the microscope is focused to a plane near the glass surface. The reason for this contrast is as follows. LC molecules are birefringent, with ordinary $\left(n_{o} \sim 1.5\right)$ and extraordinary $\left(n_{e} \sim 1.6-1.7\right)$ refractive indices. The spatial variation of the director translates into the varying refractive index mismatch between the birefringent sample and glass $\left(n_{\text {glass }} \sim 1.52\right)$. The local structure reflects less light (i.e., appears darker) when molecules are oriented perpendicularly to the glass plates, because the light experiences the ordinary index of refraction $n_{o} \sim 1.5$, which is close to the refractive index of glass. On the other hand, the structure appears brighter in reflection, when the molecules are parallel to the glass surface and there is large refractive index mismatch between the extraordinary index of the LC and glass.

This leads to a conjecture that the dark spots are halfskyrmionic director profiles forming close to the surface. In the center of the half-skyrmion, the LC molecules are oriented perpendicularly to the glass, reflecting less light due to better index matching with the glass. As we show in numerical simulations in Fig. 10(e), the half-skyrmionic profiles are indeed formed near the surfaces and are wedged 
between five to seven disclinations that attach perpendicularly to the surface in both thin and thicker BPIII.

By taking images focused to different distances from the glass-LC interface, the dark spots are indeed best resolved in a reflection, when the focal plane of the microscope is at the glass-BPIII interface. This is in agreement with our picture of DTCs emerging from the glass surface into the bulk BPIII. We also find that the optical contrast of the image is highest when illuminated with RHC-polarized light, matching the right-handedness of the chiral dopant CB15. This indicates that the image is formed of light reflected from chiral objects, which are of the same chirality as the handedness of the illumination light.

The dark spots shown in Fig. 4(c) appear in all the highresolution images of BPIII. Their optical contrast increases as we cool the mixture through the BPIII phase from the isotropic phase. Close to the BPI-BPIII transition, the dark spots are visibly fluctuating - it looks as if the spots are created and then, in the next moment, disappear, suggesting a very dynamic BPIII phase; see Supplemental Video 1 [48]. This is clear confirmation that the slow fluctuating mode observed in DLS experiments in the BPIII phase indeed corresponds to fluctuations of the DTCs.

\section{SKYRMION PHASES IN ULTRATHIN LAYERS OF BPIII LIQUID CRYSTAL}

It is practically impossible to study the structure of these dark spots, which turn out to be the surface end points of skyrmion filaments in thin layers, in several-micron-thick BPIII samples. The structure is very dynamic and fluctuating, and the optical contrast is quite low to moderate. To reveal the true nature of the dark spots in BPIII, we focus our investigations to the BPIII mixture confined to very thin layers, i.e., down to the layer thickness of approximately $50 \mathrm{~nm}$. The reasoning is as follows. If the constituents of the BPIII are the DTCs (or skyrmion filaments), they should transform into isolated, stable, and well-resolved skyrmions or DTCs in very thin layers. In general, confinement of the BP phases to layers with thickness comparable to the size of its basic constituents should reveal their form.

To this aim, we measure the thickness-temperature $(d, T)$ phase diagram of our BPIII mixture in wedge cells with variable thickness from approximately 30 to approximately $500 \mathrm{~nm}$ with degenerate planar anchoring. We measure local thickness of the cell at predetermined positions, which makes it possible to know the thickness at the selected position across the cell. The temperature of the cell is controlled and measured to better than $\pm 2 \mathrm{mK}$. The structure at a given thickness and temperature is identified by direct observation using the diffraction-limited optical microscope in reflection.

Figures 5(a)-5(d) are photographs of typical structures, observed at different thicknesses and temperatures of the BPIII mixture, whereas the resulting $(d, T)$ phase diagram is presented in Fig. 5(e). The dark spots in Fig. 5(a), taken at a thickness of the cell of only approximately $80 \mathrm{~nm}$, look exactly the same as in our previous study of BPI [23] and could immediately be recognized as the centers of isolated half-skyrmions.

We can clearly distinguish four different phases of skyrmion. (i) Isolated half-skyrmions are stable at $d<$ $80 \mathrm{~nm}$ and are shown in Fig. 5(a). This phase of diluted skyrmions (i.e., the gas phase) is observed in very thin parts of the sample and at rather low temperatures. (ii) At slightly thicker samples $80 \mathrm{~nm}<d<150 \mathrm{~nm}$, a dense phase of half-skyrmions is observed and is shown in Fig. 5(b). It is the liquidlike phase of half-skyrmions that is always positionally disordered, and we never observe a hexagonal half-skyrmion lattice in this particular BPIII mixture. (iii) At thickness $d>150 \mathrm{~nm}$, a bulk BPIII phase is observed and is shown in Fig. 5(c). (iv) The crystalline BPI phase is observed in the same range of thickness as the BPIII phase, i.e., $d>150 \mathrm{~nm}$, and is shown in Fig. 5(d). There is a first-order phase transition line between the BPIII and BPI phases, terminating around the critical thickness of approximately $150 \mathrm{~nm}$, indicated by the blue continuous line in Fig. 5(e).

Each black cross in the $(d, T)$ phase diagram in Fig. 5(e) represents an image taken. There are several interesting features of this phase diagram. First, the blue line is a BPIIIBPI phase boundary that is of first order at large thicknesses. Most importantly, this line ends in a critical point at the critical thickness of approximately $150 \mathrm{~nm}$, where both BPIII and BPI phases equally transform into the phase of dense half-skyrmions. Second, the red line is a first-order phase boundary between the BPI and the cholesteric phase that ends in another triple point, where the BPI, skyrmion, and cholesteric phases coexist. Finally, below the thickness denoted by the thin dotted line at approximately $50 \mathrm{~nm}$, no structures can be observed. This indicates the region of completely unwound chiral nematic phase, and no skyrmions are stable below this thickness.

The $(d, T)$ phase diagram of BPIII mixture reveals that skyrmions are the basic building blocks of both BPIII and BPI phases in this material. BPIII and BPI are observed in thicker parts of the sample, down to the critical thickness of approximately $150 \mathrm{~nm}$, where the skyrmion structures make a crossover from 3D to $2 \mathrm{D}$ [dashed vertical line in Fig. 5(e)]. By comparing the images in Figs. 5(a)-5(c) and the phase diagram in Fig. 5(e), we see convincing evidence that the basic constituents of both the BPIII and BPI phases are skyrmion filaments and that the BPIII is a disordered, dense phase of skyrmion filaments.

While dark spots on microscope images are identified as skyrmions, the question is why $-1 / 2$ disclinations are not visible in any of the images taken in either bulk or very thin layers of BPIII. For example, individual skyrmions are well seen in Fig. 5(a), but there is no evidence of two $-1 / 2$ disclination lines, which compensate the winding of the skyrmion. This question is already discussed and answered 

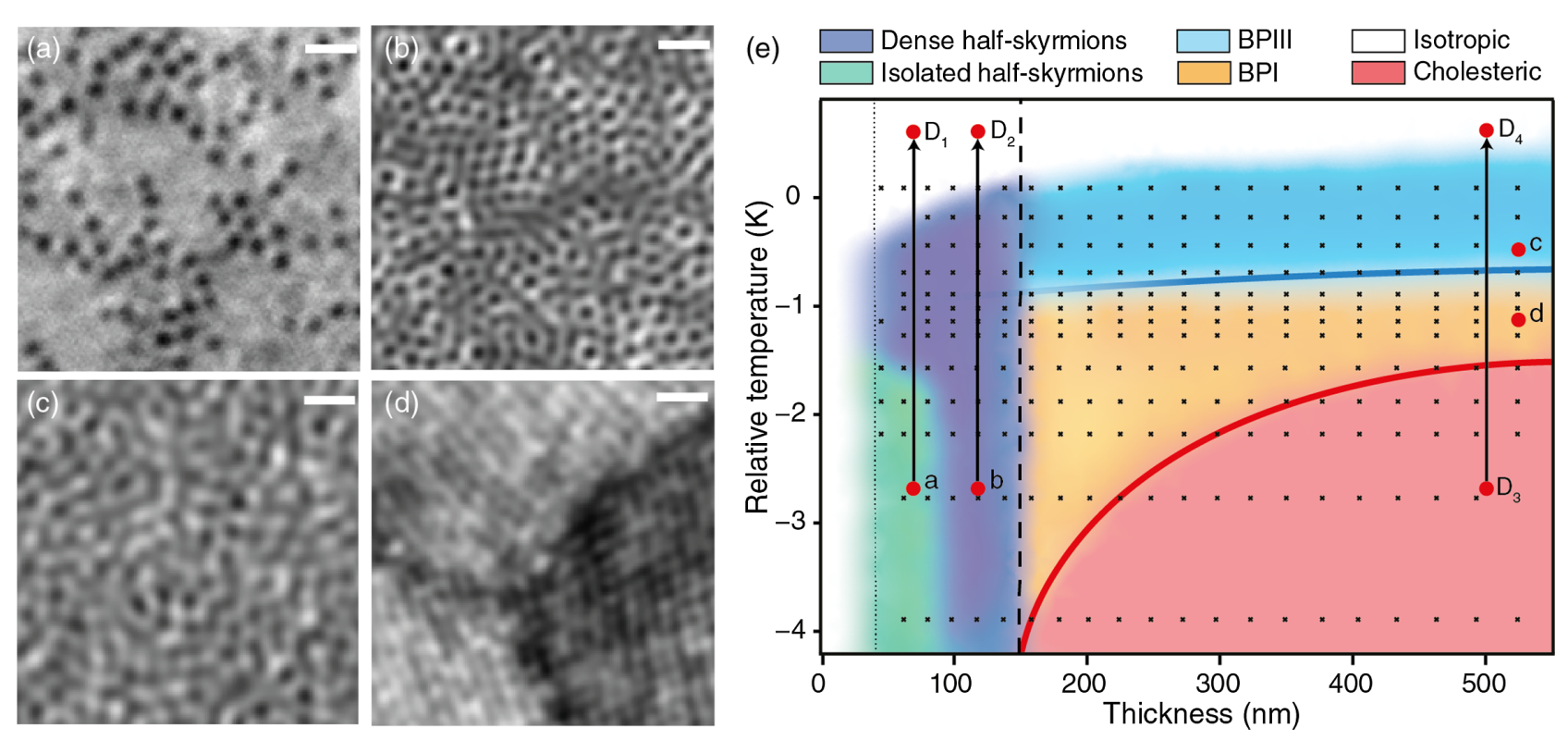

FIG. 5. Skyrmion phases in thin layers of a BPIII LC. (a) Isolated half-skyrmions are observed as approximately 120-nm-diameter dark spots at temperatures well below the BPIII phase for thicknesses less than approximately $80 \mathrm{~nm}$. The dark spots are the centers of half-skyrmions; the size of the spots is determined by the diffraction limit of the microscope. (b) A dense and disordered phase of halfskyrmions is observed in the thickness range approximately 80 to approximately $150 \mathrm{~nm}$. (c) BPIII is a stable phase above the critical thickness of approximately $150 \mathrm{~nm}$. (d) BPI is also stable above the critical thickness of $150 \mathrm{~nm}$. (e) The thickness-temperature $(d, T)$ phase diagram, as determined by heating the LC. Each black cross represents an image taken; the structures are identified from the images. Below the thickness denoted by the dotted line, no structures are observed. The blue line is a BPIII-BPI phase boundary that is of first order at large thicknesses. The red line is a first-order phase boundary between the BPI and the cholesteric phase that ends in a triple point, where the BPI, skyrmion, and cholesteric phases coexist. The lines $a-D_{1}, b-D_{2}$, and $D_{3}-D_{4}$ represent the temperature scans in Figs. 6(a)-6(c). Scale bars in (a)-(d) indicate $500 \mathrm{~nm}$. Images of different skyrmion phases in (a)-(d) are taken at $a-d$ positions marked on the phase diagram in (e).

in our previous work on half-skyrmions in very thin layers of BPI chiral liquid crystal [23]. It is shown that the two accompanying $-1 / 2$ disclination lines become visible when we observe skyrmions in BP material with a longer helical pitch. The two $-1 / 2$ disclination lines then appear as tiny dark spots, with the scattering cross section an order of magnitude smaller than the skyrmion itself. In shortpitch material, such as our BPIII, these tiny dark spots are too close to the dark image of the skyrmion and cannot be resolved due to diffraction of illuminating light.

\section{PHASE TRANSITIONS AND SKYRMION DYNAMICS IN ULTRATHIN BPIII LIQUID CRYSTAL}

The two relaxation modes in bulk BPIII, as seen in the DLS experiments [Fig. 2(b)] are consistent with the picture of fluctuating liquid skyrmion filaments: In the BPIII phase, the skyrmion filaments form a dynamic and strongly fluctuating liquid, which is completely disordered in terms of shape and orientation of DTCs. The slow mode corresponds to fluctuations of the position and shape of the skyrmion filaments, coupled to the disclination network, whereas the fast mode corresponds to twistlike structural fluctuations of the skyrmion filaments themselves [33]. This leads to the conclusion that the two relaxation modes should be observable in the thinnest layers of BPIII, where there is no place to accommodate more than a single layer of skyrmions. If so, the slow relaxation mode is a clear signature of skyrmions in the $(d, T)$ phase diagram.

When observed under an optical microscope, the dense phase of half-skyrmions below the thickness of $150 \mathrm{~nm}$ appears mostly static at lower temperatures (see Supplemental Video 2 [48]). By increasing the temperature, we can see stronger fluctuations in the positions of the individual half-skyrmions just below the isotropic phase (see Supplemental Video 3 [48]): Sometimes two half-skyrmions merge into one, and sometimes a half-skyrmion disappears and reappears from the homogeneous LC. Interestingly, there are still some thermal fluctuations in the region of individual and isolated skyrmions at very small thickness, $<80 \mathrm{~nm}$ (see Supplemental Video 4 [48]). When the temperature is increased, the density of skyrmions increases, and they finally melt continuously into the isotropic phase, never showing any coexistence of the isotropic and BPIII phases (see Supplemental Video 5 [48]). The isotropic-BPIII transition is, therefore, continuous down to the minimum measurable thickness of approximately $50 \mathrm{~nm}$.

To shed more light on the nature of the phase boundaries between the different skyrmion phases in the $(d, T)$ phase 


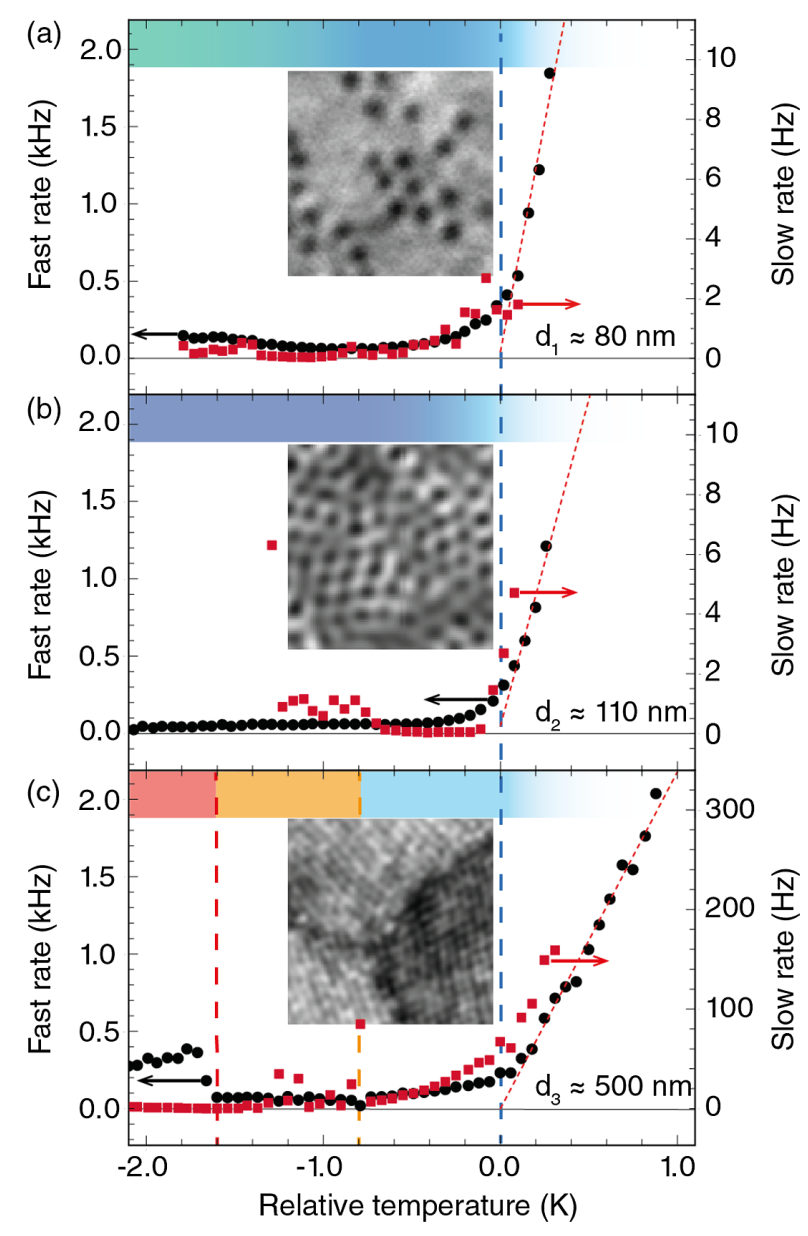

FIG. 6. Skyrmion dynamics in thin BPIII layers. (a) Temperature dependence of the relaxation rates of the fast and slow relaxation modes in approximately 80 -nm-thin layers of BPIII, measured along line $a D_{1}$ in Fig. 5(e). (b) Temperature dependence of relaxation rates in approximately 110 -nm-thin layers of BPIII, measured along line $b D_{2}$ in Fig. 5(e). (c) Temperature dependence of relaxation rates in approximately 500-nm layers of BPIII, taken along the line $D_{3} D_{4}$ in Fig. 5(e). The isotropic phase continuously evolves into BPIII, followed by BPI. The zero of the relative temperatures in (a)-(c) is determined by extrapolating the softening-mode frequency near the isotropic phase to zero.

diagram in Fig. 5(e), we explore the temperature and thickness dependence of the nematic order-parameter (OP) dynamics using cross-differential dynamic microscopy (cDDM; for details of the method, see Appendix B) in very thin layers of the sample and comparing it to the OP dynamics in bulk BPIII, obtained by DLS (Fig. 2).

In cDDM imaging, one seeks for the correlation of image details with variable time delay between the two images taken. Slow changes in the subsequent images result in longer correlation times, whereas fast changes result in shorter measured correlation times. We perform cDDM experiments using RHC-polarized light in wedge samples with regions as thin as $80 \mathrm{~nm}$ and in the temperature interval $\Delta T \sim 3^{\circ} \mathrm{C}$ around the isotropic-to-BPIII phase transition in

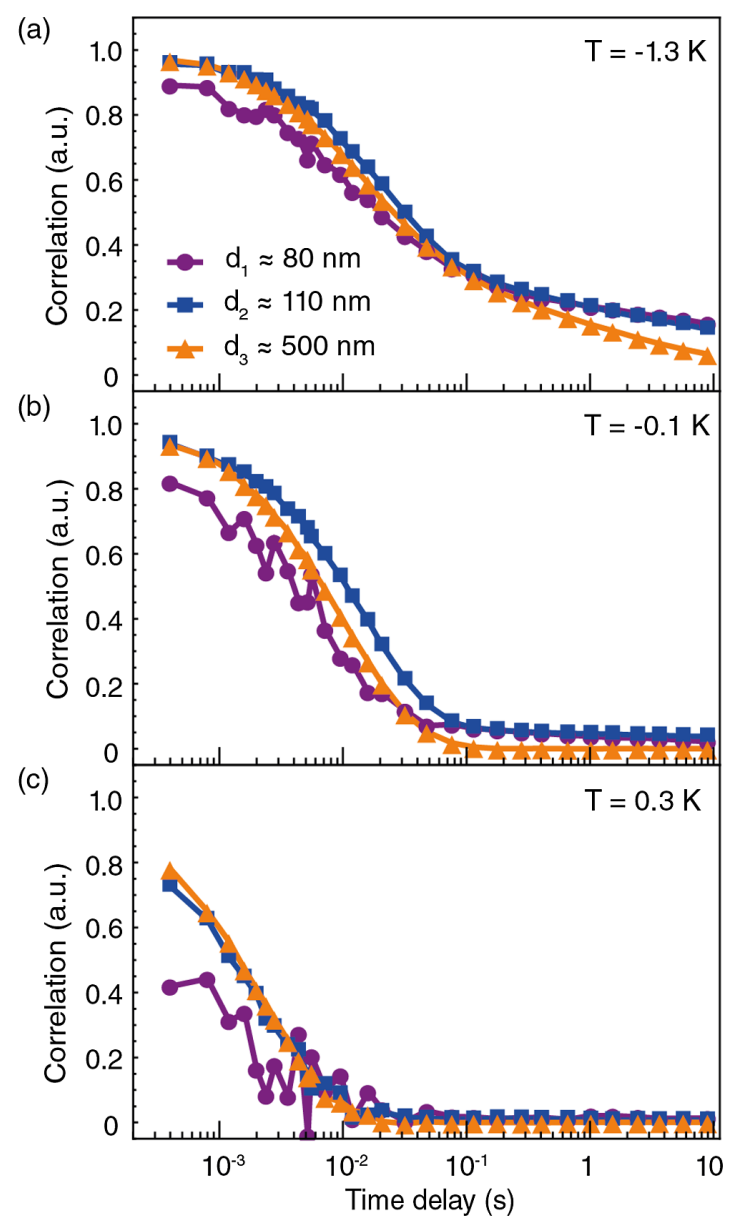

FIG. 7. Order-parameter dynamics in skyrmion phases of BPIII mixture measured by cDDM autocorrelation. (a) The dense skyrmionic phase at $T=-1.3 \mathrm{~K}$ shows a slow relaxation "tail," indicating the presence of static objects. (b) Just below the isotropic phase at $T=-0.1 \mathrm{~K}$, the slow tail in the autocorrelation functions is still observed, indicating two-mode dynamics. (c) A single relaxation mode is observed for all thicknesses in the isotropic phase at $T=0.3 \mathrm{~K}$.

the bulk. Similar to the DLS, where DTC fluctuations are strongest in the RHC-RHC scattering geometry for this RH chiral LC, changes in (chiral) skyrmion images are best seen when RHC polarization is used for illumination. The results are shown in Figs. 6(a)-6(c). For all the measured thicknesses from 80 to $500 \mathrm{~nm}$, we observe two fluctuating modes. Whereas the dynamics in the approximately 500-nm-thin layer of BPIII [Fig. 6(c)] is still like the dynamics in the bulk [Fig. 2(b)], there is a substantial change in the dynamics of the slow mode below the crossover thickness of approximately $150 \mathrm{~nm}$. At a thickness of $110 \mathrm{~nm}$ [Fig. 6(b)], the fluctuating rates of the slow mode drop from approximately $20 \mathrm{~Hz}$ in the BPIII [red squares in Fig. 6(c)] to $0.1 \mathrm{~Hz}$ [red squares in Figs. 6(a) and 6(b)].

This dramatic slowing of the skyrmion dynamics below the critical thickness of $150 \mathrm{~nm}$ is clear from the measured cDDM autocorrelation functions in Figs. 7(a) and 7(b). They 


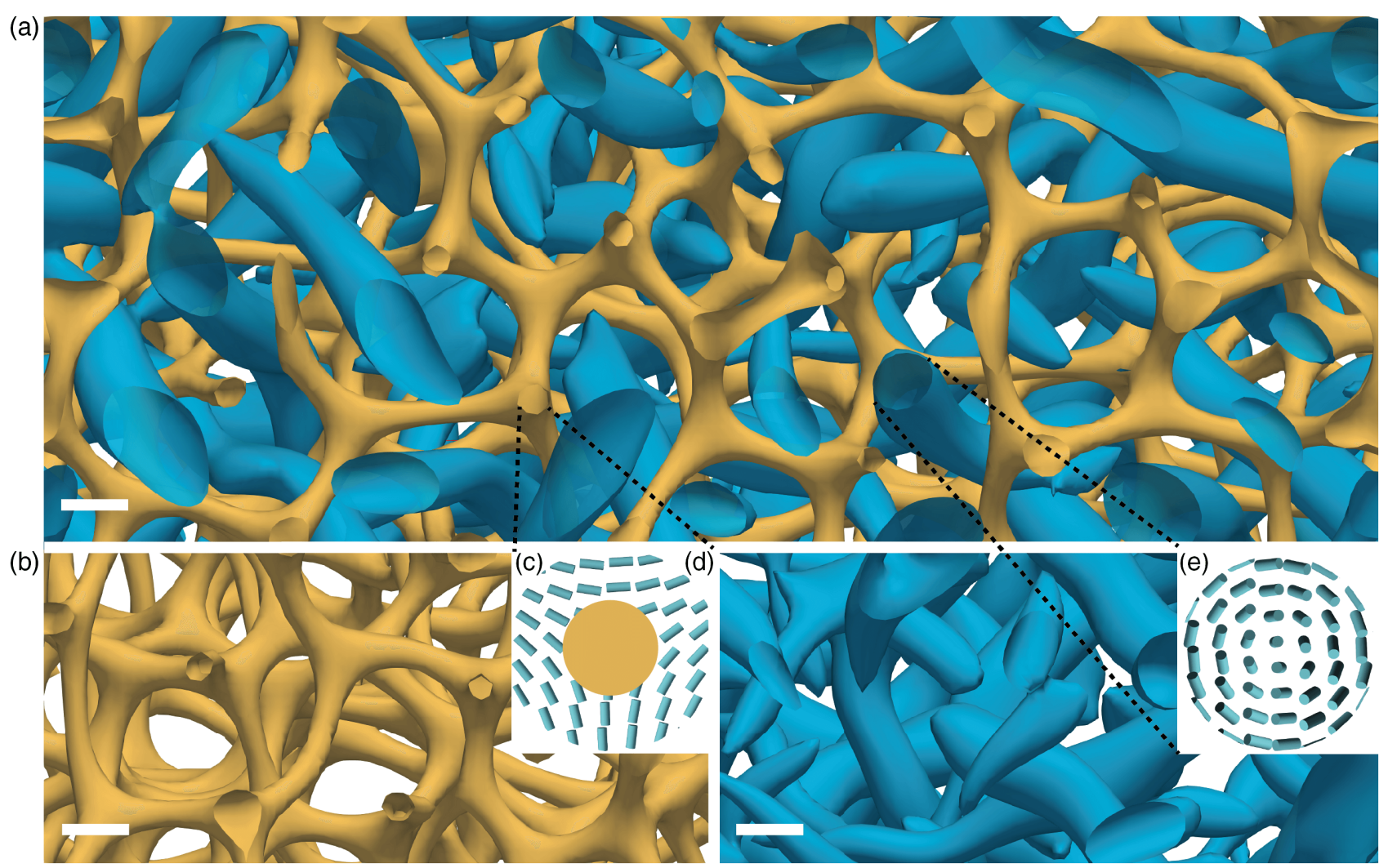

FIG. 8. Numerical simulations of bulk BPIII. (a) Bulk BPIII is a topological fluid of skyrmionic filaments (shown in blue; see Appendix C), enmeshed with a 3D network of $-1 / 2$ chiral singular lines (shown in yellow). (b) Separate view of $-1 / 2$ disclination network with $S=0.14$ [continuing from (a)]. (c) Cross section of a defect line in (b); the singular core is in yellow. (d) Skyrmion filaments [continuing from (a)] are not branched; some of them start or end at a defect line. (e) Quarter-skyrmion radial profile of a DTC in (a). Scale bars represent $50 \mathrm{~nm}$.

all obtain a long tail, which vanishes in the isotropic phase in Fig. 7(c). This tail is a clear indication of the long-lived objects, also seen on the captured images (see Supplemental Video 4 [48]), which disappear in the isotropic phase. These static objects are half-skyrmions, and their lifetime in a dilute and dense skyrmion phase is around $10 \mathrm{~s}$. It is important to note that the long tail in the autocorrelation function is a clear indication of lingering half-skyrmions on the captured images, even if they cannot be distinguished by the naked eye in the photographs of the same structures.

A comparison of Figs. 6(a)-6(c) shows how, in thinner LC samples, both the BPIII and BPI [Fig. 6(c)] transform into the same dense half-skyrmion phase in Fig. 6(b) and then further to the diluted skyrmion phase (gas) of isolated half-skyrmions in Fig. 6(a). This is another strong evidence that BPIII is a liquid of skyrmion filaments and BPI is a crystal phase of the very same constituents and is in full agreement with the static phase diagram in Fig. 5.

\section{NUMERICAL AND OPTICAL SIMULATIONS OF BPIII SKYRMIONS}

To corroborate the experiments, we perform extensive Landau-de Gennes (LdG) numerical modeling of stable and metastable skyrmion structures in thin layers and bulk
BPIII; details can be found in Appendix C. Similar to previous work by Henrich et al. [43,44], we reproduce the bulk BPIII of mutually enmeshed structures of singular defect lines and skyrmion filament-DTCs, as shown in Fig. 8(a). The network of disclinations [Fig. 8(b)] has a reduced degree of nematic order $S$ in the cores and shows $-1 / 2$ winding of the director field to compensate for the effective topological charge imposed by the skyrmion filaments [Fig. 8(c)]. The skyrmionic filaments are not branched and are free of singularities [Fig. 8(d)], with an approximately $\pi / 4$ radial twist deformation of the director field [Fig. 8(e)]. DTCs in bulk BPIII can, therefore, be considered as quarter-skyrmions, although, more precisely, there is a distribution of maximum radial twist angles of DTCs (i.e., their actual size) in bulk BPIII, as discussed in the continuation.

The simulated BPIII is thermodynamically stable and has lowest free energy only for a selected range of chirality and temperature values. This reflects a fragile balance between the elastic energy and the energy contribution due to Landau-de Gennes expansion of the free energy (see Appendix C). A typical energy difference between the amorphous BPIII and the higher-energy cubic BP in simulations is about $1 \%$ of the total free energy. 
(a)

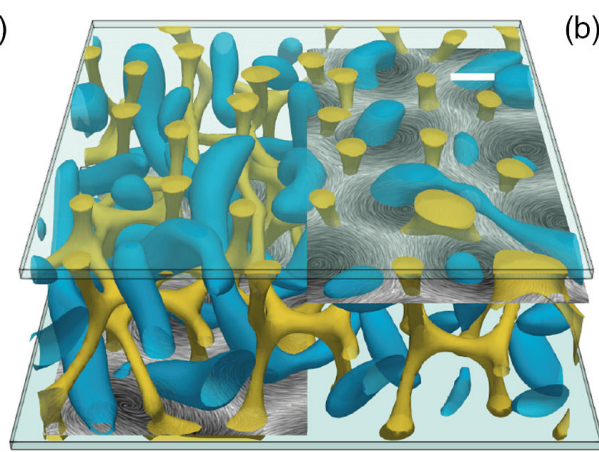

(c)

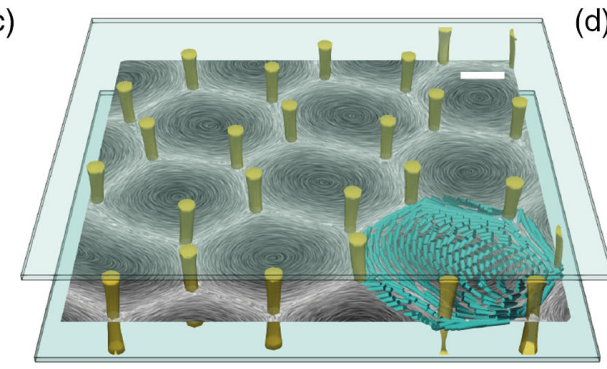

(b)

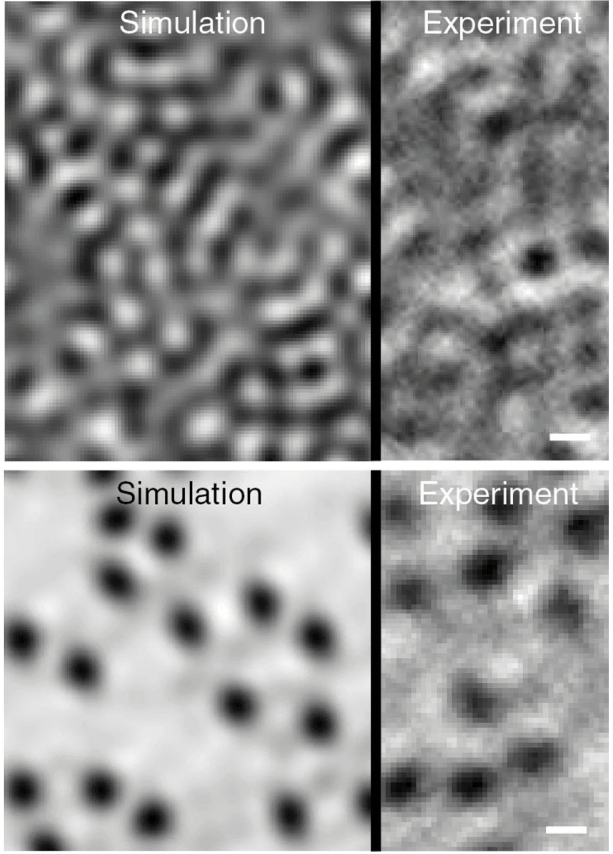

FIG. 9. Numerical and optical simulations of thin layers of BPIII. (a) Skyrmion filaments (light blue) and $-1 / 2$ defect lines (yellow) in 300-nm-thick LC with strong surface anchoring. The intertwined lattices of skyrmion filaments and defects start filling the space, forming the BPIII. (b) Optical simulation of light reflected from the structure in (a) and comparison with the experimental image of BPIII at an experimental cell of approximately $300 \mathrm{~nm}$ thickness. (c) Simulated baby half-skyrmions in 135-nm-thin layer of BPIII are vortexlike formations of the director, filling the space between $-1 / 2$ disclination lines connecting the upper and lower surfaces. (d) Optical simulation of numerically calculated half-skyrmions below the crossover thickness $(135 \mathrm{~nm})$ and comparison with experiments. The color scale of the director in (a) and (c) is from zero (white) to one (black) and denotes the out-of-plane director component. Scale bars indicate $100 \mathrm{~nm}$ on (a) and (c) and $50 \mathrm{~nm}$ in (b) and (d).

The LdG simulations of BPIII confined to thin layers (130-300 nm) with parallel surface anchoring of LC molecules are shown in Figs. 9 and 10. When confined below the crossover numerical LC thickness of approximately $170 \mathrm{~nm}$, quarter-skyrmion filaments of the bulk BPIII [Fig. 8(a)] disentangle into a 2D, disordered, half-skyrmion liquid [Fig. 9(c)]. The numerical crossover thickness of approximately $170 \mathrm{~nm}$ is in remarkable agreement with the experimental value of approximately $150 \mathrm{~nm}$, which gives confidence to our calculations of skyrmion structures in thin layers of BPIII. It is important to note that, because of the confinement and parallel surface anchoring, quarter-skyrmions in the bulk liquid BPIII transform to half-skyrmions with an approximately $90^{\circ}$ radial twist. These baby half-skyrmions [Fig. 9(c)] fill the space between the $-1 / 2$ disclination lines: Usually, a single half-skyrmion is formed in the region surrounded by five to seven vertical $-1 / 2$ disclinations that connect both confining surfaces.

At a slightly larger thickness, elongated skyrmion filaments start to grow preferably at an oblique angle to the confining surfaces, as shown in Fig. 9(a). Simultaneously, the individual $-1 / 2$ defect lines start connecting with each other laterally [Fig. 9(a)], forming a network of disclinations with junctions of four $-1 / 2$ lines fused together [53]. This intertwined network of skyrmion filaments and $-1 / 2$ disclination lines at large thickness evolves into a network of fully disordered, 3D structure of skyrmion filaments and defects of the bulk, as shown in Fig. 8(a). It is interesting to note that the skyrmion structures close to the surfaces of the cell all remain very similar for all cell thickness, which is due to the dominating surface alignment field. Consequently, this produces similar optical images in thin and thick layers, when focused near the surface, where reflection images are shown to originate from.

To compare the optical appearance of simulated skyrmion structures and real photographs, we make full optical simulations of various skyrmion structures, which are shown in Figs. 9(b) and 9(d). The simulations for different handedness of illuminating light show that right-handed $(\mathrm{RH})$ skyrmion structures provide a better optical contrast with the RHC-polarized illumination, which is in full agreement with the experiments. The overall agreement between the recorded and simulated images is excellent.

The director field in an approximately 130-nm-thin layer of BPIII is presented in Figs. 10(a) and 10(d) and shows the $-1 / 2$ defect lines (colored yellow) standing up vertical from the bottom glass surface (colored light blue) and connecting to the upper glass surface. 

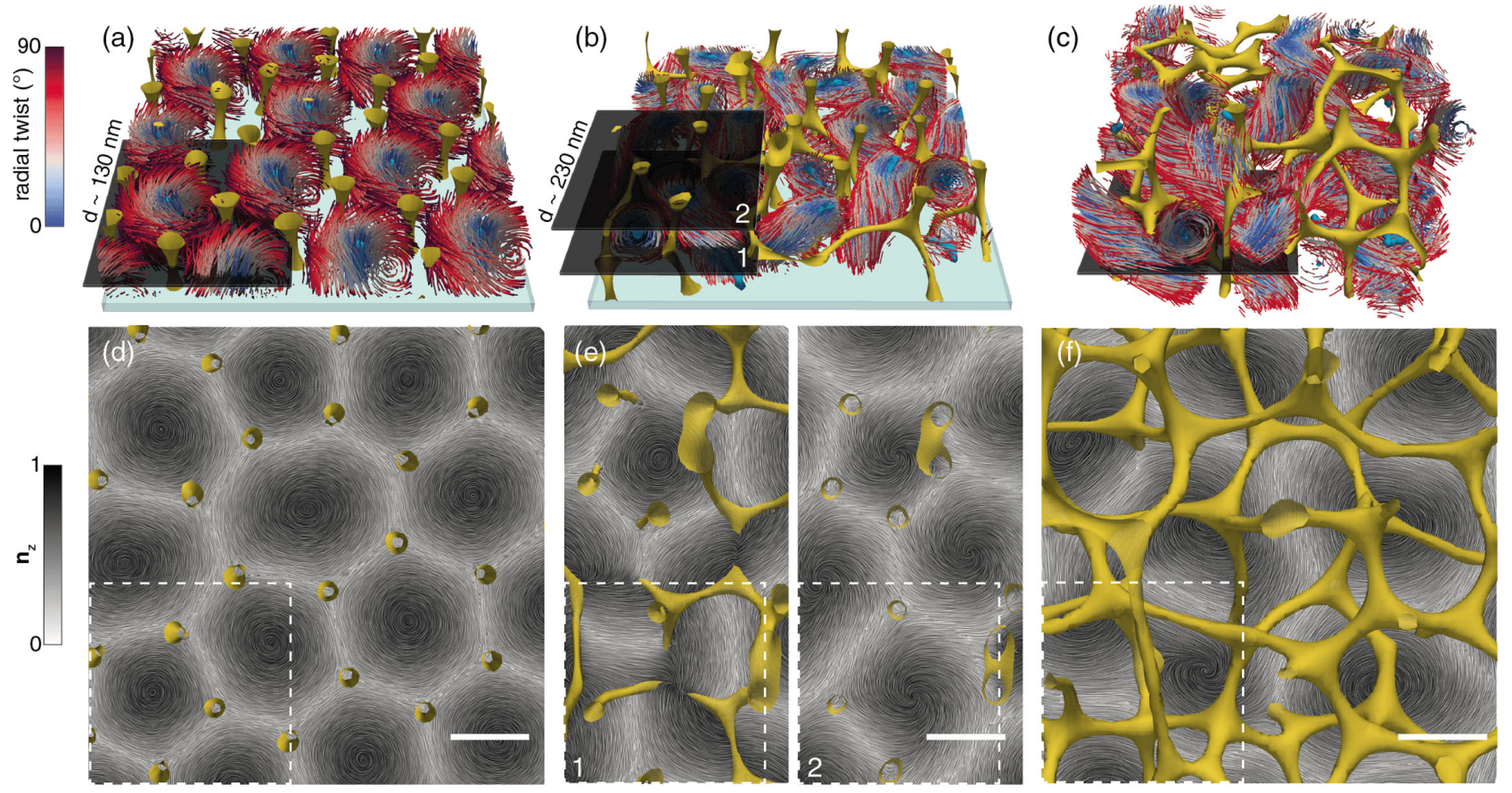

FIG. 10. Director field in BPIII skyrmion structures. (a) The simulated director structure of half-skyrmions in an approximately 130nm-thin BPIII layer. Colored light blue is the bottom glass plate of the cell. The upper glass plate is not shown for clarity. (b) The simulated director structure of skyrmions in an approximately 230-nm-thick layer. (c) The simulated director structure of skyrmions in bulk BPIII. BPIII skyrmions are visualized using vector field lines colored according to the local radial twist with respect to the center of the skyrmion. The color bar for the radial twist is shown on the left. (d) The director field in the cross section marked by the dark plane in (a), which is located at the center of the cell. The dashed box corresponds to the dark plane in (a). (e)(1) shows the skyrmion director structure in the middle of an approximately 230-nm-thick BPIII layer shown in (b), marked by dark plane 1 . The dashed box corresponds to dark plane 1 in (b). (e)(2) shows the director at the interface to the upper glass in (b), marked by dark plane 2 . The dashed box corresponds to dark plane 2 in (b). (f) Director field in bulk BPIII, indicated by the dark plane in (c). The dashed box corresponds to the dark plane in (c). The director field in (d)-(f) is shown with streamlines, colored black, where the director points out of plane and white, when it is in plane in accordance with the color bar on the left. In all images, the singular disclination lines are shown in yellow. Scale bars correspond to $100 \mathrm{~nm}$.

The director streamlines are colored according to the radial twist, with red streamlines indicating approximately $90^{\circ}$ twist and blue streamlines indicating $10^{\circ}-20^{\circ}$ twist with respect to the core of the vortex. It is clearly seen from the dominating red-colored streamlines in Fig. 10(a) that the skyrmions in an approximately 130-nm-thin layer of BPIII are half-skyrmions developing large, approximately $90^{\circ}$ radial twist. A vertical view of the same approximately $130-$ nm-thin layer of BPIII is presented in Fig. 10(d). Here, the view is taken in the middle of the BPIII layer, and the gray scale indicates the $z$ component of the director, which is perpendicular to the glass surface. Vortices are now clearly seen, and the dark center indicates that the molecules are aligned perpendicular to the glass surface in the center of half-skyrmions. When moving outward from the center of the skyrmion, the streamlines become white at the periphery, indicating near-parallel alignment of LC molecules with the surface. The skyrmions are clearly disordered, in both position and size.

Figure 10(b) shows director streamlines in an approximately 230-nm-thin layer of BPIII, which is above the
2D-3D crossover thickness of approximately $170 \mathrm{~nm}$. Although the streamlines are still red colored, indicating approximately $90^{\circ}$ radial twist of vortices, one can clearly see the emergence of $-1 / 2$ disclination lines, which are running parallel to the surface, in addition to disclination lines, which connect both surfaces. This is clearly seen from the director streamlines taken in two planes in Fig. 10(b). Figure 10(e)(1) shows the midcell view of director streamlines, showing vortices and also $-1 / 2$ disclination lines (yellow) connecting to vertical $-1 / 2$ disclination lines along the midplane of the BPIII layer. Figure 10(e)(2) shows near-surface director streamlines and $-1 / 2$ disclination lines that attach perpendicularly to the surface of glass. Finally, we present in Figs. 10(c) and 10(f) director streamlines and network of mutually connected $-1 / 2$ disclination in bulk BPIII. A cross section through bulk BPIII in Fig. 10(f) clearly shows vortices, which are enmeshed with $\mathrm{a}-1 / 2$ disclination network.

This analysis indicates the trend of changes in the director structure, when bulk BPIII is confined to thinner and thinner layers. When the BPIII layer is thinner than a 


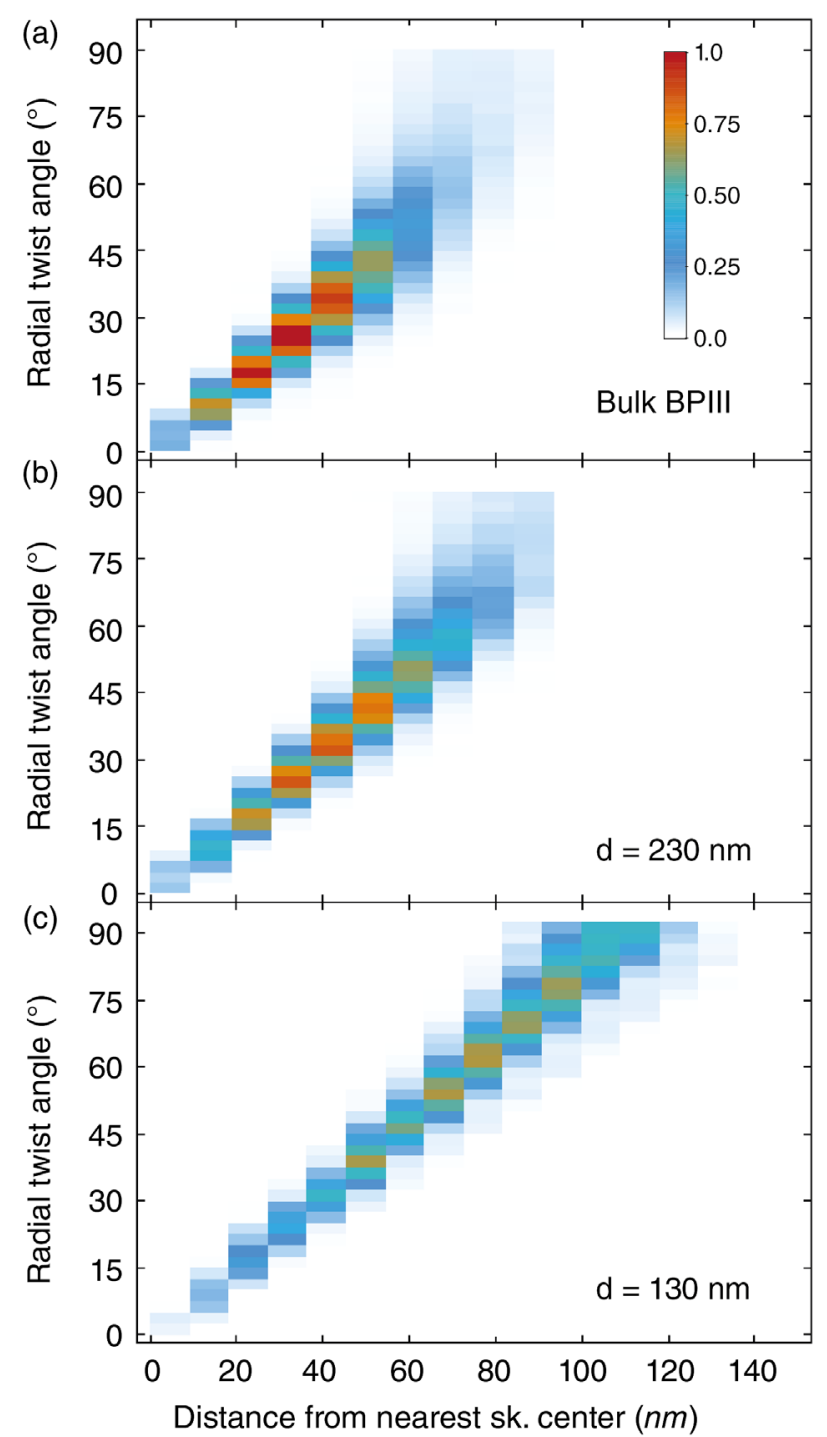

FIG. 11. Radial director twist angle distribution in thin, thick, and bulk BPIII. (a) The distribution of radial director twist for all voxels as a function of the distance from the skyrmion center, calculated for bulk BPIII in Fig. 10(c). (b) The distribution of radial director twist for all voxels as a function of the distance from the skyrmion center, calculated for 230-nm-thick BPIII in Fig. 10(b). (c) The distribution of radial director twist for all voxels as a function of the distance from the skyrmion center, calculated for 130-nm-thin BPIII in Fig. 10(a).

single skyrmion layer of $170-n m$ thickness, it cannot accommodate any $-1 / 2$ disclination line, except those which connect both surfaces directly. The tangle of bulk BPIII vortices, therefore, disentangles into a thin layer of dense and well-resolved skyrmions, which confirms our conjecture that confinement should reveal the basic constituents of the BPIII phase.

While it is clear from the director profiles in a very thin approximately $130-\mathrm{nm}$ layer of BPIII that vortices are actually half-skyrmions with $90^{\circ}$ twist, it is less clear from tangled skyrmion structures at larger thickness what kind of skyrmions are there. To understand this, we analyze radial twist angles for bulk BPIII and 230- and 130-nm-thin layers of BPIII. A computer program recognizes the centers of vortices and for each nearby voxel determines the value of radial twist with respect to the nearest vortex center. The results are then visualized as a distribution, where the $x$ axis is a distance from the center of the nearest skyrmion, the $y$ axis is the radial twist in that voxel, measured from the nearest skyrmion center, and the color map measures the normalized number of voxels that have that particular twist at that particular distance. Distributions of radial twist for bulk and 230- and 130-nm-thin layers of BPIII are shown in Fig. 11. Details about the methodology of skyrmion radial twist analysis can be found in Appendix D.

In bulk BPIII, the skyrmions are definitely not halfskyrmions with up to approximately $90^{\circ}$ radial twist, as can be seen from the radial twist distribution in Fig. 11(a). The radial twist of skyrmions in bulk BPIII is not well developed, as the distribution hardly includes voxels with approximately $60^{\circ}$ radial twist. The distribution in bulk BPIII shows a peak at approximately $30^{\circ}-35^{\circ}$ radial twist, indicating the DTCs are not highly radially twisted.

When the bulk BPIII is confined to 230-nm-thin layers, the distribution of radial twist broadens toward higher twists, as shown in Fig. 11(b). Finally, in 130-nm layers of BPIII (which is below the critical thickness of $170 \mathrm{~nm}$ ), the distribution of radial twist further expands to up to $90^{\circ}$ director twist, which is shown in Fig. 11(c). From the distributions of radial twist in Fig. 11, one can clearly recognize the trend of increasing radial twisting of DTCs, as the BPIII material is confined. Whereas in bulk BPIII the skyrmions are practically fractional skyrmions with approximately $35^{\circ}$ radial twist, they are forced to twist

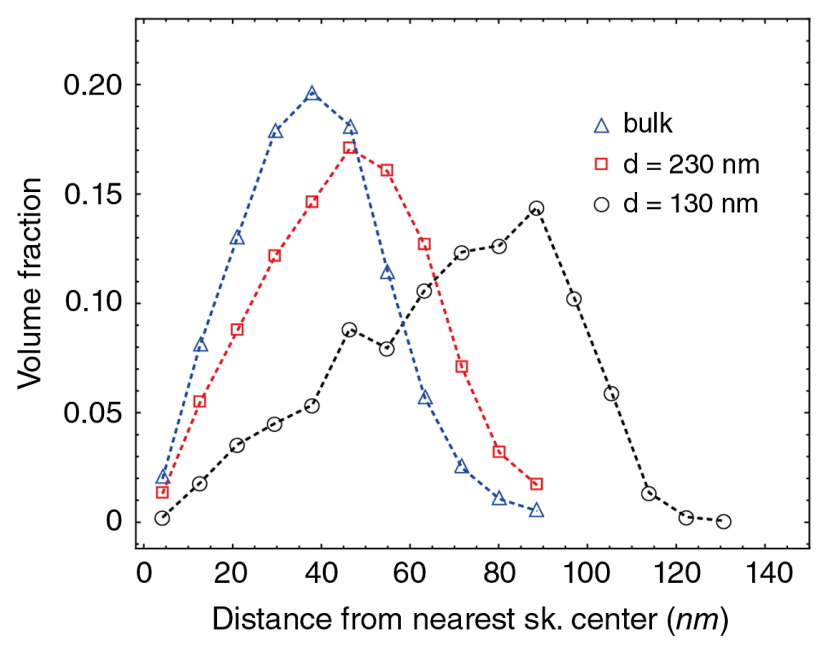

FIG. 12. Diameter of DTCs in bulk and confined BPIII. Volume fraction of all voxels in the simulated chiral structures as a function of the distance from the nearest skyrmion center for thin, thick, and bulk BPIII. The peak corresponds to an effective radius of the skyrmion filament. 
under confinement and transform into half-skyrmions with approximately $90^{\circ}$ radial twist in 130 -nm-thin layers.

The effective diameter of skyrmion filaments (i.e., the DTCs) can be determined by plotting the volume fraction of voxels attributed to a given skyrmion. This fraction grows linearly up to the radius $R$ of the DTC and then starts falling, because the voxels further away belong to another, neighboring DTC. Figure 12 shows this volume fraction of voxels belonging to a given skyrmion for bulk BPIII and 230- and 130-nm-thin BPIII. In bulk BPIII (triangles in Fig. 12), the diameter of a single DTC is approximately $80 \mathrm{~nm}$. When BPIII is constrained to thin film, the effective diameter of DTCs increases to approximately $100 \mathrm{~nm}$ (squares in Fig. 12) and further on to approximately $180 \mathrm{~nm}$ in 130-nm-thin BPIII. This is consistent with the increasing value of radial twist upon confinement of BPIII. The more radial twisting angle of a DTC, the larger the diameter of the DTC.

\section{DISCUSSION}

We present experiments and numerical simulations, which show that the BPIII of chiral liquid crystals is a dynamical tangle of double-twist filaments (skyrmion filaments) and disclination defect lines, which, when confined to very thin layers, disentangle into a 2D liquid of half-skyrmions. There are three important emerging points to be discussed here.

First, the dynamical nature of BPIII, observed even with the naked eye under a diffraction-limited optical microscope, raises the question as to the nature of the isotropicBPIII phase transition and the associated order parameter. In our BPIII material, this unusual phase transition is clearly continuous, as we never observe any sign of the coexistence of the isotropic and the BPIII phase. Moreover, the optical contrast of BPIII fluctuations increases continuously as we cool the sample from the isotropic to the BPI phase. Our dynamic light scattering and image crosscorrelation experiments clearly demonstrate two different relaxation modes in bulk BPIII, the relaxation rates of which differ for one order of magnitude. Most importantly, the slow fluctuations on the scale of $100 \mathrm{~ms}$ and longer are attributed to the spatial motion and reorientation of wellformed double-twist filaments. On the other hand, it is clear from DLS measurements that the fast mode exists in bulk BPIII, which slows down and condenses in the Landau sense at the phase transition from BPIII to BPI. We therefore have two different phase transition temperatures, which are correlated by the underlying physics. The BP soft mode condenses at BPIII to BPI phase transition temperature, breaks the symmetry of the isotropic phase, and condenses into a crystal BPI phase. On the other hand, while the symmetry of the isotropic phase is not yet broken, a slow mode occurs at the isotropic-BPIII transition, because the skyrmion filaments are definitely formed and fluctuate. Clearly, the mean field Landau-type theory
[37] has to be considered in terms of the relevant order parameter to describe the fast and slow fluctuations during the symmetry breaking across the isotropic-BPIII-BPI phase transitions.

Second, our numerical simulations reveal that a BPIII cannot be directly considered a liquid of half- or full skyrmions with approximately $90^{\circ}$ and approximately $180^{\circ}$ radial twist, respectively. Instead, we show that BPIII skyrmions are actually fractional (i.e., even real-number) skyrmions with around $35^{\circ}$ radial twist, which is significantly lower than the intuitively expected value of $45^{\circ}$.

Third, there is a puzzling stability of skyrmion filaments in BPIII. Although individual skyrmion filaments are not stable when immersed into a homogeneous and infinite background orientational field, an ensemble of skyrmion filaments attains stability against thermal disruption, as evidenced by the experiments. The system gains a collective property that we could describe as "collective topological protection," as provided by the network of disclinations. From a topological perspective, this work opens questions about fundamental topological charge compensation in systems with general-disordered and interconnected-networks of topological defects and structures, as far beyond the current knowledge on usually pairwise compensation of topological charge by distinct topological objects (or defects).

We note that the BPIII is in some aspects similar to the nonequilibrium state of active turbulence in 3D that has recently been explored both theoretically [54-56] and experimentally [57]. In BPIII, topological defects are generated by the chirality of the liquid crystal that spontaneously twists the LC in $2 \mathrm{D}$, whereas in active nematics [58] topological defects are generated by the self-amplified bend instability fueled by the external supply of the energy to the system. It is shown in a numerical analysis of $3 \mathrm{D}$ active nematic confined to a droplet [56] that above some energy threshold the system transits into the state of active turbulence, where the topological defects are continuously generated, annihilated, merged, and split or reconnected. It is also shown that in 3D the majority of defects are closed zero topological charge loops that imply conservation of the total topological charge of the system. We note that the state of 3D active turbulence enjoys a similar sort of collective topological protection, where the topological charge is constant in time-as if in an effective steady state-despite regular dynamic topology affecting events, such as rewiring, merging, generation, and annihilation of defect loops. In BPIII, the collective topological protection is ensured by the strong chirality of the material, which energetically prevents the system from transit into a homogeneous state without defects.

Because the BPIII is an enmeshed and disordered 3D structure of skyrmion filaments and a mesh of $-1 / 2$ defect lines, it is an open challenge how to count the topological charge of its elements [53]. The BPIII mesh of singular 
defects is fully connected, but, since in all defect vertices one observes an even number of joining defect line segments, could it be effectively topologically decomposed into a mesh made of closed loops, which then touch in the vortices? Indeed, in 3D active nematics, the defect lines appear to be mainly loops [57], which we speculate could possibly transform into an active defect mesh upon introducing chirality into the active nematic system [59].

Since the structure of BPIII is amorphous, the actual thickness of DTCs and the maximum radial twist of different skyrmion filaments both vary throughout the sample. The effective diameter of a particular skyrmion filament crucially depends on the local environment imposed by other filaments and the disclination network. In accordance with the soft nature of a LC orientational field, boundaries between neighboring chiral skyrmions and disclinations are not sharply defined, which further suggests that their fractional topological charge can be thought of as a continuously distributed space-varying property as well.

The numerically calculated effective size of the skyrmionic filaments in thick BPIII is approximately $80 \mathrm{~nm}$ (for $270 \mathrm{~nm}$ chiral nematic pitch) and is, therefore, considerably below the optical resolution limit of a standard, diffraction-limited optical microscope using visible light. Our comparison of experimental and theoretical optical images, as well as the calculated structures, suggests that the experimental images of BPIII textures are a diffraction-limited view of fractional skyrmion configurations formed near the confining glass plates. In a thinner region, the contrast variation is a diffraction-limited view of a half-skyrmion itself, which have a larger diameter of approximately $180 \mathrm{~nm}$. This size of half-skyrmions is just at the limit of the optical resolution of a diffraction-limited optical microscope in LCs [60]. This means that the dark spots in Fig. 9(d) (right) are indeed photographs of single half-skyrmions.

Our work has clear implications for other fundamentally different condensed matter systems, distinctly including the quantum blue fog in quantum helimagnets. Quantum blue fog is a magnetic analog of BPIII phase of liquid crystals as proposed by Tewari, Belitz, and Kirkpatrick [61] to explain the unusual $(p, T)$ phase diagram of $\mathrm{MnSi}$. At ambient pressure, $\mathrm{MnSi}$ exhibits a continuous phase transition at $30 \mathrm{~K}$ into the helically modulated magnetic phase (helimagnet) with a helical period of $17 \mathrm{~nm}$, observable by neutron scattering. By increasing the pressure, the paraferro phase transition gradually becomes first order, and the phase transition temperature drops to the critical temperature at a critical pressure of approximately $12 \mathrm{kbar}$. For higher pressure, a new phase is observed, which shows some spectacular non-Fermi-liquid (NFL) behavior. Within the NFL region, neutron scattering experiments give evidence that NFL has a short-range helical order but is disordered on a larger scale. It can, therefore, be considered as either a chiral gas or chiral liquid, respectively. Tewari, Belitz, and Kirkpatrick propose that the NFL phase is actually composed of a higher-temperature chiral gas phase and low-temperature chiral liquid phase, separated by a phase transition line in the $(p, T)$ diagram. They predict that chiral magnetic gas phase could be analogous to the chiral isotropic phase of BPIII LCs and the chiral liquid phase could be an analog of the BPIII phase of LCs. The phase transition between chiral gas and chiral liquid is then analogous to gas-liquid condensation first proposed by Lubensky and Stark for BPIII [37].

We propose to test the validity of quantum blue fog theory by measuring the order parameter dynamics across the NFL region of MnSi. Should the blue fog be a chiral liquid, then two relaxation mode dynamics must be observed in this phase, similar to the two relaxation mode dynamics in our BPIII measurements. Dynamics of the order parameter has actually been measured in $\mathrm{MnSi}$ by neutron spin echo spectroscopy [62], and a dynamically disordered magnetic phase has been observed at ambient pressure. The order parameter relaxation times are around one nanosecond at the phase transition into the helical phase, which is most likely the soft mode relaxation and not a signature of the quantum blue phase.

\section{PERSPECTIVES AND CONCLUSIONS}

Magnetic skyrmions were proposed as basic bits of information and have been intensively studied for application in information storage and logic technologies [63]. Magnetic skyrmions are topologically protected whirls of magnetization, which are stable in thin films of chiral magnetic materials, provided that an external magnetic field is applied. At cryogenic temperatures, their dimensions are as small as few nanometers, and they can be created, annihilated, and transported by spin-polarized electric currents. One of the major obstacles for practical applications of magnetic skyrmions is their stability at room temperature. Furthermore, in room-temperature skyrmionic materials, the diameters of magnetic skyrmions are rarely below $100 \mathrm{~nm}$, and the dispersion of skyrmion sizes is a serious problem [19].

On the other hand, BP skyrmions are of comparable size to magnetic skyrmions at room temperature. In our BPIII material, the size of skyrmions is around $90 \mathrm{~nm}$ at a cell thickness of $130 \mathrm{~nm}$. The fact that individual skyrmions in our BPIII material are dynamic indicates they could be created and annihilated using strongly focused LaguerreGaussian beams, as demonstrated by Ackerman [15] for larger, micrometer-sized full skyrmions. It has also been recently demonstrated by Smalyukh's group [64] that full skyrmions can be moved in a controlled manner by applying an external electric field of variable frequency. Furthermore, the information could be encoded in BP skyrmions by incorporating fluorescent dye molecules, which align with the local director. The fluorescent 
molecules act as polarized light sources, and the intensity of light emitted from these molecules depends strongly on their orientation [65]. When excited, fluorescent dye molecules generate strong fluorescence that can be easily detected regardless of skyrmion size. Because of alignment of fluorescent dye molecules within the whirling structure of a skyrmion, fluorescence emission from individual BP skyrmions could easily be discriminated from the neighboring homogeneous LC.

One of the most important findings of this work is the temperature range of stability of single and dense skyrmions below the critical thickness of $150 \mathrm{~nm}$, as presented in Fig. 5(e). Whereas the temperature range of both BPIII and BPI phases is around $2 \mathrm{~K}$ at large thickness, we see that the temperature range of dense half-skyrmions is more than doubled to approximately $4 \mathrm{~K}$. This broadening of halfskyrmion phase under confinement is very important for realization of practical skyrmion-based devices.

Finally, we address the problem of the size of individual half-skyrmions, which is around $90 \mathrm{~nm}$ in our BPIII mixture (Fig. 11). The size of a skyrmion scales with the chirality of the BP material, and, in order to make BP skyrmions smaller, LC materials with stronger chirality have to be used. We comment that BP materials with much shorter helical pitch and, therefore, much higher chirality have already been studied and used in LCDs. For example, we have studied short-period cholesteric LCs using AFM [66] and stimulated emission depletion microscopy [60]. The cholesteric pitch is as short as $150 \mathrm{~nm}$ in a mixture of BL087 (commercial cyano-biphenyl mixture, Merck KGaA, Germany) and a chiral dopant R5011 (Merck KGaA, Germany). This pitch is much shorter than the pitch of $225 \mathrm{~nm}$ in the cholesteric phase of our BPIII mixture. Most probably, the skyrmion size (if they are stable in BL087/R5011) is expected to be around $50 \mathrm{~nm}$, which will be addressed in future study. To obtain LC skyrmions with sizes below $10 \mathrm{~nm}$, new and highly chiral LC materials have to be developed.

In conclusion, our measurements provide convincing experimental evidence that BPIII is a topologically protected liquid of strongly fluctuating skyrmion filaments with special dynamics. The basic building units of BPIII are the skyrmion filaments, enmeshed with 3D branched tangles of $-1 / 2$ disclination lines. Together, they form a highly dynamic, topologically protected liquid of oppositely topologically charged entities, which cannot be annihilated due to the elastic energy barrier. This work not only provides a compelling structure of BPIII, it also points to the possible realization of a quantum blue fog in quantum helimagnets, it opens questions on the nature and order parameter dynamics at topological phase transitions in soft ordered matter, and it opens questions on the countability of topological charge in disordered topological systems with vertices of defect lines. From the aspect of possible applications, we give convincing evidence that blue phase skyrmions are quite competitive and, in some aspects, superior to magnetic skyrmions.

\section{ACKNOWLEDGMENTS}

I. M. thanks Ivan I. Smalyukh for discussion on the occasion of the Colloidal Mikado workshop in Oxford that inspired this study. We acknowledge discussions with Junichi Fukuda at early stages of this work. We also acknowledge the support and discussions with S. Žumer and Ž. Kos. This work was supported by Slovenian Research Agency (ARRS) through Contracts No. P1-0099 (I. M.), No. PR08332 (J. P.), No. J1-1697 (M. M.), No. J1-2462 and N10195 (M. R.) and No. P1-0192 (A. Pet. and A. M.).

\section{APPENDIX A: MATERIALS AND SAMPLE PREPARATION}

\section{BPIII liquid crystal, sample preparation, and optical microscopy}

The experiments are performed in a 1:1 wt $\%$ mixture of bent-core liquid crystal (5-3CF3-2M-5) [67] and righthanded chiral liquid crystal CB15 [(S)-4-(2-Methylbutyl)4'-cyanobiphenyl, Merck KGaA] and is designated in the text as BPIII mixture. The mixture is prepared for the experiments by first heating it above approximately $90^{\circ} \mathrm{C}$, which is the temperature of the isotropic phase transition of the component 5-3CF3-2M-5 LC. The mixture is kept at this temperature for a couple of hours and mixed regularly using a Vibromix vortex shaker to ensure good mixing of the two components. Using a Cano wedge method, we estimate the unit cell size $a$ of the BPI to approximately $215 \mathrm{~nm}$, which is close to the value of the helical pitch in the cholesteric phase at lower temperatures.

To prepare 30-500-nm-thin layers of BPIII mixture for microscopic observations and cDDM measurements, a small amount $(0.2-0.5 \mu \mathrm{L})$ of the prepared mixture is pressed between two cover glass plates. We use 150- $\mu \mathrm{m}$ thick cover glass plates (VWR catalog No. 48366-089, $25 \mathrm{~mm} \times 25 \mathrm{~mm}$ ) cleaned with ethanol and used immediately after alcohol evaporates. This type of glass provides planar surface anchoring of our BPIII mixture. After pressing a droplet between the glass plates, the cell is held at an elevated temperature $\left(60^{\circ} \mathrm{C}\right)$ for approximately 30 min to spread and equilibrate. Once the LC spreads between the two glass surfaces, the resulting wedge cell usually has a varying thickness from tens of nanometers to a few microns. We should note that the choice of cover glass plates is crucial to obtain very thin layers of a LC, and using this type of cover glass even 50-nm-thin LC could be made.

The wedge LC sample is first inspected under a microscope using an $x-y$ motorized stage (Prior ES107). Then, a line across the wedge is selected, along which the LC material shows appropriate variation of the local thickness from tens of nanometers to a micrometer. Local cell 
thickness at predetermined positions along the chosen line is determined by recording the reflection spectra of the thin LC layer in the isotropic phase. The spectra are measured using an Andor Shamrock SR-500i-D1 spectrometer equipped with an Andor Newton EM DU970N camera. The spectra at each stored position are then fitted using the model spectra with local LC layer thickness as a free parameter. This makes it possible to calculate local thickness of the LC layer at all predetermined positions, which could be relocated during the experiments using the stored coordinates of the $x-y$ motorized stage.

Color images of LC textures and optical reflection spectra from thick BP samples are taken on an upright Nikon Eclipse E600 microscope operating in reflection mode between crossed polarizers. The sample is placed in a thin and rectangular glass capillary placed on a heater at a position where a temperature gradient of a few degrees is present between the two ends of the capillary. Temperature dependence of the spectra of white light reflected from the approximately $20-\mu$ m-thick cell filled with BPIII mixture are recorded using an Ocean Optics USB2000 spectrometer, mounted on an upright Nikon Eclipse E600 microscope and illuminated with an incandescent light bulb. Sample temperature is controlled using an Instec HCS302 heater stage and Instec STC200 controller.

Black and white, diffraction-limited microscope images and movies are recorded using an inverted optical microscope (Nikon Ti-U) operating in reflection mode.The microscope is installed in a temperature-controlled room with better than $1 \mathrm{~K}$ temperature stability. When performing longer temperature scans, the room is vacant and the experiment is remotely controlled. To realize the diffraction-limited resolution, a high numerical aperture oil immersion objective with $100 \times$ magnification is used (CFI Plan Apo Lambda 100× Oil, NA 1.45). To minimize chromatic abberations, the sample is illuminated by a narrow band light blue $(450 \mathrm{~nm})$ LED (CoolLed LED pE-300). Some images are taken without polarizing elements inserted using an Andor Neo camera with 6.5- $\mu \mathrm{m}$ sized pixels on a cooled sensor and an additional $2.5 \times$ magnification. These images are passed through a lowpass Fourier filter, to remove the high-frequency image noise. Sample temperature for optical microscopy and cDDM measurements is controlled using a custom-made microscope sample heater and a Wavelength Electronics PTC2.5K-CH temperature controller with temperature stability better than $5 \mathrm{mK}$.

\section{APPENDIX B: EXPERIMENTAL METHODS}

\section{Temperature calibration of different setups}

A number of different measurement techniques are employed in our experiments, which use different experimental cells and different experimental setups. This requires a method of cross calibration of temperature scales to accurately identify the phase transition temperatures, as obtained from different experiments. We use the isotropicnematic phase transition of 4-cyano-4'-pentylbiphenyl (5CB, Merck, KGaA) as a reference temperature point. $5 \mathrm{CB}$ is a high-purity single-component LC with a welldefined first-order phase transition from the isotropic to the nematic phase. We measure the temperature of the firstorder nematic-isotropic phase transition in the 5CB samples in different setups and use it as a temperature reference point for all measurements. This approach makes it possible to put all the experimental results from different measuring methods on the same temperature scale with an accuracy of the order of approximately $0.1 \mathrm{~K}$.

\section{Optical rotation}

We use ORP measurements to determine the isotropicBPIII phase transition temperature in our BPIII mixture. This transition is continuous in our mixture and is, therefore, difficult to determine by microscope observations. However, ORP provides a good method to determine the temperature of this phase transition, as shown in a number of experiments [27,33]. In ORP experiments, we measure the optical rotation of a linearly polarized light propagating through a $140-\mu \mathrm{m}$ layer of a BPIII mixture. We use a standard experimental setup [68] based on an optical photoelastic modulator (PEM-90, Hinds Instruments), which modulates the phase retardation of light with a frequency of $50 \mathrm{kHz}$ and uses a dual lock-in amplifier detection to minimize the effects of the uncontrolled light-intensity fluctuations. The optical rotation angle $\Psi$ is determined by the ratio of the first $\left(U_{1}\right)$ and second $U_{2}$ harmonic of transmitted light $\Psi=1 / 2 \tan ^{-1}\left(k U_{1} / U_{2}\right)$, where $k$ is the ratio of Bessel functions at a constant amplitude of retardation defined by the modulator. The wavelength of the transmitted light is $632.8 \mathrm{~nm}$, and the resolution of the phase retardation measurement is $0.01^{\circ}$. The measurements of optical rotation are obtained during slow cooling from the isotropic through both blue phases with the cooling rate of $1 \mathrm{~K} / \mathrm{h}$.

DLS is a well-established method to measure the dynamical properties of liquid crystals [69], and many DLS studies have been performed in BP liquid crystals in the past $[32,33,50,51]$. DLS is a far-field optical technique that yields ensemble-averaged information on the dynamics of the system from nanoseconds to second relaxation times, with the wave vector of the fluctuations depending on the laser and scattering geometry used.

Our experiments are performed in a standard setup [32,33,50,51], using a He-Ne laser $(632.8 \mathrm{~nm}, 20 \mathrm{~mW})$, an avalanche photodiode based "pseudo" cross correlation detector, and ALV-6010/160 correlator to obtain the autocorrelation function of the scattered light intensity. The sample is placed in a rectangular glass capillary (thickness 
$50 \mu \mathrm{m}$, width of the capillary $1 \mathrm{~mm}$ ). The angle between the wave vector of RHC-polarized incoming light and the normal to the sample plane is $-14^{\circ}$, and the angle between the wave vector of the detected and incoming light is $140^{\circ}$. An attenuator of 0.7 is used to reduce the intensity of the incoming light. The RHC analyzer is placed in front of the detector. A single-mode optical fiber with a graded-index lens is used to collect the scattered light within one coherence area.

We fit the intensity autocorrelation function $g_{2}$ to $g_{2}=1+2\left(1-j_{d}\right) j_{d} g_{1}+j_{d}{ }^{2} g_{1}{ }^{2}$, where $j_{d}$ is the ratio between the intensity of the nonelastically scattered light and the total scattered intensity. $g_{1}$ is either a single exponential function (in the I phase), $g_{1}=a e^{-t / \tau}$, or a sum of a single and a stretched exponential function $g_{1}(t)=a e^{-\left(t / \tau_{1}\right)^{s}}+(1-a) e^{-t / \tau_{2}}$. The capillary is placed in a heating stage (Instec HCS412W) and controlled by temperature controller (Instec, mK2000) to better than $5 \mathrm{mK}$.

\section{Cross-differential dynamic microscopy}

While DLS is very powerful method to analyze dynamical properties of LCs in bulk samples, some attempts have been made to use it in combination with an optical microscope [70-73]. In our experiments, we are interested in the dynamics of topological objects in very thin layers of liquid crystal, which are, in fact, similar to colloidal systems. A number of different methods based on optical microscopy have been developed to study dynamics of colloidal systems [74,75], which are optical near-field techniques. We therefore adopt a variant of differential dynamic microscopy (DDM) [74], which uses an optical microscope with incoherent white light illumination in combination with digital video recording of images.

The experiments are performed by using a modified DDM setup, named cDDM, as described by Arko and Petelin [76]. The setup consists of a relay lens system, followed by a beam splitter and two identical cameras (Flir BFS-U3-162M-CS) with $3.45 \mu \mathrm{m}$ pixel size and 1/3-inch sensor. The setup is equipped with translation and rotation mechanisms that allow for a very precise alignment of their field of view. To reduce the light-heating and degrading effects of the illuminating light to the LC sample, we use strong LED pulses of the yellow (approximately $550 \mathrm{~nm}$ ) colored LED of the CoolLed pE-300 illumination system for cDDM measurements. For some measurements, a leftor right-handed circular (RHC and LHC, respectively) polarizer is inserted into the optical path immediately after the light source, creating nearly circularly polarized light at the sample plane. The ratio between the semiaxes of the polarization ellipse is approximately $1.1: 1$ for the used yellow light.

The light which is backreflected from the LC is collected using the same high-NA objective that is used for illumination. The collected light continues its way into the cDDM setup through the mounting lens $(0.7 \times)$ microscope port, which allows a larger field of view and access to higher wave-vector projections. Using a mechanical alignment system, the two imaging arrays of two identical cameras are brought into identical positions with respect to the image of the LC sample. Each of the two cameras is triggered randomly and independently to acquire two different sets of images. The typical exposure time for image acquisition is $100 \mu \mathrm{s}$, and the shortest (nonzero) time delay between two consecutive frames taken on different cameras is set to $400 \mu$ s. Typically $2^{15}$ or $2^{16}$ frames are acquired in approximately $15 \mathrm{~min}$ during a single measurement of the cross-correlation. Such a two-camera setup is not limited by maximal frame acquisition rate of a single camera and allows us to probe sample dynamics at rates up to $3 \mathrm{kHz}$ using this LC sample and pulsed LED illumination.

Using an open-source package for cDDM [77], the acquired image sequences are cross Fourier analyzed as a function of the time delay between the frames, and normalized image correlation functions are calculated at all achievable wave-vector projections simultaneously. For data presented in Figs. 6(a)-6(c), a subset of autocorrelation functions taken at a wave-vector projection around $18 \mu \mathrm{m}^{-1}$ are averaged, and the autocorrelation functions are fitted using $g(t)=a e^{-\left(t / \tau_{1}\right) s}+(1-a) e^{-t / \tau_{2}}+b$. Here, $\tau_{1}$ and $\tau_{2}$ describe the fast and slow correlation decay modes, respectively. The effective relaxation rate of the stretched fast mode is calculated using $f_{1}=s / \Gamma(1 /|s|) \cdot 1 / \tau_{1}$, where $\Gamma$ is the Gamma function [78]. Using the cDDM technique, the dynamics of skyrmions could be measured in samples as thin as approximately $50 \mathrm{~nm}$. The main limitation of measuring the dynamics in even thinner samples is the very low intensity of the light, reflected from the LC sample and collected by the two cameras.

\section{APPENDIX C: NUMERICAL SIMULATIONS}

\section{Numerical modeling of bulk and confined blue phase III structures}

Bulk and confined blue phase III are modeled by using continuum Landau-de Gennes free energy approach, based on the full order parameter tensor $Q_{i j}[79,80]$. This approach is especially strong for modeling chiral nematic structures at the mesoscopic scale, which exhibit singular topological defects and textures, such as the blue phases. The liquid crystalline director $\boldsymbol{n}$ is then determined by the eigenvector of $Q_{i j}$, corresponding to its maximal eigenvalue, given by the nematic degree of the order of $S$. The Landau-de Gennes total free energy $F$ of chiral nematic LC is 


$$
\begin{aligned}
F= & +\int_{\mathrm{LC}}\left\{\frac{A_{0}(1-\gamma / 3)}{2} Q_{i j} Q_{j i}-\frac{A_{0} \gamma}{3} Q_{i j} Q_{j k} Q_{k i}\right. \\
& \left.+\frac{A_{0} \gamma}{4}\left(Q_{i j} Q_{j i}\right)^{2}\right\} d V \\
& +\frac{L}{2} \int_{\mathrm{LC}}\left\{\left(\frac{\partial Q_{i j}}{\partial x_{k}}\right)^{2}+\left(\epsilon_{i k l} \frac{\partial Q_{l j}}{\partial x_{k}}+2 q_{0} Q_{i j}\right)^{2}\right\} d V \\
& +\frac{1}{2} W \int_{\text {surface }}\left(Q_{i j}-Q_{i j}^{0}\right)\left(Q_{j i}-Q_{j i}^{0}\right) d S .
\end{aligned}
$$

Here, LC denotes an integral over the volume of chiral nematic LC. The top row gives the bulk free energy of nematic and describes a change of the free energy due to changes in the nematic degree of the order of $S$ (such as in the cores of the defects), $A_{0}$ is a material constant, and $\gamma$ an effective dimensionless temperature. The middle row gives the free energy cost of elastic distortions in the liquid crystalline order. We use the one-elastic-constant approximation, where $L$ is a single elastic constant. $q_{0}$ is the wave vector of spatial modulation of $Q(\boldsymbol{r})$ and is related to the pitch of the cholesteric helix $\left(p=2 \pi / q_{0}\right)$. $\epsilon_{i j k}$ is the Levi-Civita fully antisymmetric tensor. The bottom row presents surface contributions. For modeling of surface anchoring at the top and bottom surface, we use planar degenerate surface free energy as introduced in Ref. [81] with local degree of order (i.e., in each simulation point) in the transformation of the order parameter tensor.

For bulk simulations, the free energy is minimized using an explicit Euler finite difference algorithm on a cubic mesh with periodic boundary conditions in all three directions. In thin cells, we use periodic boundary conditions in two directions, and we set degenerate planar surface anchoring at the top and bottom surface [81]. Both limits of strong and weak anchoring are considered in simulations and produce similar structures in the BPIII parameter regime. We fix the number of points in the simulation to 32 points per chiral pitch, which gives sufficient resolution at reasonable resources. This gives the mesh resolution of $8.4 \mathrm{~nm}$ for a chiral pitch of $p=270 \mathrm{~nm}$, which is a fair approximation of the experimental conditions, where the lattice constant of BPI is measured at approximately $215 \mathrm{~nm}$. The following parameter values, characteristic for the chiral nematic used in experiments, are set: material constant $A_{0}=$ $1.02 \times 10^{5} \mathrm{~J} / \mathrm{m}^{3}$, elastic constant $L=2.5 \times 10^{-11} \mathrm{~J} / \mathrm{m}$, and anchoring strength $W=7 \times 10^{-3} \mathrm{~J} / \mathrm{m}^{2}$. By comparing the free energy of BPI, BPII, O5 (see Ref. [82]), cholesteric, and BPIII structures in a wide range of chirality-temperature phase space, we find that the BPIII is the energetically favorable structure (i.e., has the lowest free energy) in a narrow range of high chiralities and temperatures near the nematic-isotropic transition. For most of the results presented in this paper, we use the dimensionless temperature $\gamma=3.0$.
The structures shown in this article are a result of the numerical relaxation of a $Q$-tensor field. Typically, $N=$ 100000 iterative (time) steps are needed in thin cells, whereas up to 10 million time steps are needed in bigger and in bulk samples. For generation of bulk blue phase III textures, we use a similar approach as in Ref. [43], which can bias the selection of a given chiral structure by proper selection of initial conditions. In bulk samples, cholesteric, BPI and BPII and O5 structures are achieved using appropriate analytical expressions for the initial condition, which define the symmetry of the structure and the periodicity [43]. On the other hand, BPIII is an amorphous phase and has to be initiated from a nonsymmetric initial state. Specifically, the BPIII disordered structure is achieved by initiating the system in the isotropic phase with a low density (typically $2 \%$ volume fraction) of randomly placed and randomly oriented quarter-skyrmion spherical regions. The initialization of the BPIII is not strongly dependent on the exact volume fraction of the quarter-skyrmion spherical regions. We observe that already one or two quarter-skyrmion spherical regions ( $0.2 \%$ volume fractions) are enough to initialize the formation of BPIII structure. The BPIII structure evolves from these cores to the whole sample typically in a few thousand time steps, with the actual number of time steps depending on the number of these skyrmion cores and the size of the simulation box. Using $2 \%$ volume fraction gives enough quarter-skyrmion cores to reliably get BPIII-like structure even in a range of simulation volumes. A smaller volume fraction slightly increases the relaxation time. For $0 \%$ volume fraction, the system remains in the isotropic phase.

The same initial condition for BPIII is used in confined geometry of a planar cell. In the parameter regime, where BPIII is the global free energy minimum in bulk simulations, the relaxed structure is typically not periodic, even if the size of the simulation box allows for only approximately $5 \times 5$ skyrmions to form. Randomly placed halfskyrmion regions oriented normal to the surface are used as initial conditions in very thin planar cells to produce halfskyrmions. These spontaneously grow to form the thin BPIII structure at the BPIII parameter regime.

To study the BPIII structures at different cell thickness, we gradually increase the thickness of the cell, ranging from the thinnest cell of approximately $100 \mathrm{~nm}$ up to approximately $500 \mathrm{~nm}$, as used in the experiments. For each thickness, the structure is initiated from the final state of a slightly thinner structure, interpolated to a bigger mesh, and relaxed to find the new equilibrium.

\section{Visualization of DTCs from numerically calculated BPIII structures}

For visualization of the structure of BPIII in Figs. 8-10, the $-1 / 2$ topological defects are presented as an isosurface of scalar order parameter of $S=0.14$, whereas the 
skyrmion filaments are visualized as the light blue isosurface of a visualization parameter $\Phi$. This parameter has a local minimum in the center of the skyrmion filaments (DTCs). Interestingly, the visualization parameter $\Phi$ is directly related to an effective calculation of an electric potential as obtained from minimizing the (free energy) functional for $\Phi$ in the form [83]

$$
\begin{aligned}
F= & \pm \int_{\mathrm{LC}}\left\{G\left(\frac{\partial Q_{i j}}{\partial x_{i}}\right)\left(-\frac{\partial \Phi}{\partial x_{j}}\right)\right\} d V \\
& \pm \frac{1}{2} \int_{\mathrm{LC}} \epsilon_{0} \epsilon_{i j}\left(\frac{\partial \Phi}{\partial x_{i}}\right)\left(\frac{\partial \Phi}{\partial x_{j}}\right) d V .
\end{aligned}
$$

Here, $G$ can be seen as the flexoelectric coefficient in the single flexoelectric constant approximation, $\epsilon_{0}$ is the dielectric vacuum permittivity constant, and $\epsilon_{i j}$ is the dielectric permittivity tensor. In the physical interpretation of the visualization parameter $\Phi$, the first contribution describes the flexoelectric effect, whereas the second contribution is the free energy of the dielectric interaction. It should be stressed that this parameter $\Phi$ correctly visualizes DTCs in BPI and BPII phases and is therefore adopted to visualize the DTCs in BPIII bulk and confined structures as well.

\section{Diffractive transfer matrix and finite-difference time-domain simulations}

To interpret real microscope images in terms of calculated director structures, real images are compared to optically simulated images, where the director structures are taken from numerical calculations. To this aim, we build a simplified virtual microscope model that includes the actual illumination and light collection details. In optical microscopy, one uses a Koehler illumination setup, which produces uniform illumination from a nonuniform light source. This setup maps each point of the spatially incoherent light source to a single uniform plane wave. The spatial position of each of the point source defines the incidence angle of the uniform plane wave. The condenser aperture defines the maximum incidence angle and the numerical aperture of the illumination. The so-called illumination cone produced by the illumination setup can be treated as a sum of noninterfering plane waves with wave vectors evenly distributed inside the cone with an opening angle defined by the condenser aperture. There are an infinite number of plane waves inside the cone in real microscopes, but in simulations, we define a finite set of plane waves, with wave vectors carefully chosen to match the experiment's boundary conditions. Because we use periodic boundary conditions in light propagation simulations, we simulate the illumination with a finite set of eigenwaves propagating in different directions in the sample's cover glass.
To compute the EM field at the object plane under the illumination conditions described previously, we use an open-source package for light propagation called the diffractive transfer matrix method (DTMM) [77]. DTMM is a vectorial FFT-based split-step beam propagation method, similar in concept to the FFT-based beam propagation method introduced in Ref. [77], where the material is treated as a series of layers comprising of a thin lens, followed by a homogeneous layer. In contrast to the method by Feit and Fleck [84], the DTMM algorithm is vectorial and bidirectional and computes the Fresnel reflections at the interfaces, allowing one to track the reflected waves by iteratively passing the field multiple times in both the forward and backward directions through the sample. The algorithm is exact for homogeneous layers and is accurate for low-birefringence and low-contrast samples with slowly varying optical properties. Note that the director structure in each layer of the sample is taken from corresponding numerical LdG calculations.

After double passing through the sample, the microscope objective collects the reflected light within a collection cone with an opening defined by the numerical aperture of the objective and reconstructs the near-field focusing plane EM field at the imaging plane on the detector. In our simulations, we use a simplified approach and treat the objective as a thin lens that performs a linear transformation of the object plane field using a point-spread function defined by the lens's opening aperture. Thus, we can simulate the lens's finite numerical aperture. To simulate the focal plane shift, we first propagate the field from the object plane to the focal plane. Solving the Helmholtz equation in the Fourier space allows us to simulate the focal plane shift by multiplying the mode coefficient with a proper phase factor. Therefore, to simulate the image formation of the objective lens, we first compute the reflected field for each of the illumination plane waves at a given object plane above the sample. Because this field is an EM field propagating in a homogeneous medium (the cover glass), we can perform mode decomposition using Fourier transform to obtain a set of forward and backward propagating eigenwaves. For image reconstruction, this allows us to remove the incidence field from the computed EM field and take only the backward propagating (reflected) field. We then perform focal plane readjustment and simulate the finite numerical aperture of the objective. From the reconstructed EM field, we then compute the intensity. We repeat the computation for all illumination plane waves. The total intensity is a sum of the intensities obtained from each of the single plane wave illuminations.

To test the validity of the results obtained from the DTMM calculation, we also perform more accurate finitedifference time-domain (FDTD) calculations using Meep [85] and also our own developed FDTD package [86]. The simulation results show a good qualitative match between DTMM and FDTD simulated images using RHC polarized 
light. For both DTMM and FDTD simulations, we use the same $Q$ tensor structure. The nematic birefringence is set to $\Delta n=0.11$, with $n_{o}=1.57$ and $n_{e}=1.68$. We place the structure between two glass layers with $n=1.52$. The wavelength used is $\lambda=450 \mathrm{~nm}$. In FDTD, we collect the computed $\boldsymbol{E}$ and $\boldsymbol{H}$ field at a distance of about 1.5 wavelengths from the glass-structure interface to collect only the propagating modes of the EM field (with no evanescent fields). Because of the Yee lattice used in FDTD simulations, the $\boldsymbol{H}$ and $\boldsymbol{E}$ data are displaced in time and space. We use Meep's interpolation scheme to compute the $\boldsymbol{H}$ and $\boldsymbol{E}$ fields in the Yee lattice center, which allows us to do a proper plane wave decomposition and image reconstruction. In FDTD simulations, we limit the source light's incidence angle to an angle of $45^{\circ}$, which results in a $N A_{\text {ill }}=1$, whereas, in DTMM, we could simulate higher values of $N A_{\text {ill }}$. A more detailed comparison of DTMM and FDTD simulated images of BPIII skyrmion structures in thin layers will be presented in a separate publication.

\section{APPENDIX D: DISTRIBUTION OF RADIAL TWIST IN SKYRMIONS}

To show that the blue filaments represented in Figs. 8-10 are, in fact, fractional skyrmion filaments, we analyze the LdG simulated data and calculate the statistics of distribution of the radial twist angle of the director relative to the distance from the skyrmion centers. A numerical algorithm recognizes the centers of the skyrmion filaments employing both the local director orientation $\boldsymbol{n}(\boldsymbol{r})$ and the parameter $\Phi$ [Eq. (C2)]. This produces a smooth surface defining the center of the skyrmion filament [represented with light blue isosurfaces in Figs. 8(a) and 8(c)], where the director is aligned tangentially to the surface. After the skyrmion filaments are recognized, each voxel in the entire simulation volume is assigned to the nearest skyrmion and then all of the voxels are distributed to bins, based on (i) the distance to the nearest skyrmion center and (ii) the radial twist, i.e., the angle the director makes relative to the director orientation in the nearest skyrmion center.

Since the skyrmions are cylindrical objects, the radial twist should grow linearly from the skyrmion center outward. This can be seen in Figs. 11(a)-11(c), which show the distribution of radial director twist for three different numerically calculated skyrmion structures presented in Figs. 10(a)-10(c), respectively.

[1] T. H. R. Skyrme, A Unified Field Theory of Mesons and Baryons, Nucl. Phys. 31, 556 (1962).

[2] M. Rho and I. Zahed, The Multifaceted Skyrmion (World Scientific, Singapore, 2016).

[3] N. D. Mermin and T.-L. Ho, Circulation and Angular momentum in the a Phase of Superfluid Helium-3, Phys. Rev. Lett. 36, 594 (1976).
[4] S. E. Barrett, G. Dabbagh, L. N. Pfeiffer, K. W. West, and R. Tycko, Optically Pumped NMR Evidence for Finite-Size Skyrmions in GaAs Quantum Wells near Landau Level Filling $v=1$, Phys. Rev. Lett. 74, 5112 (1995).

[5] A. E. Leanhardt, Y. Shin, D. Kielpinski, D. E. Pritchard, and W. Ketterle, Coreless Vortex Formation in a Spinor Bose-Einstein Condensate, Phys. Rev. Lett. 90, 140403 (2003).

[6] U. K. Rössler, A. N. Bogdanov, and C. Pfleiderer, Spontaneous Skyrmion Ground States in Magnetic Metals, Nature (London) 442, 797 (2006).

[7] S. Mühlbauer, B. Binz, F. Jonietz, C. Pfleiderer, A. Rosch, A. Neubauer, R. Georgii, and P. Boni, Skyrmion Lattice in a Chiral Magnet, Science 323, 915 (2009).

[8] L. Wang, Q. Feng, Y. Kim, R. Kim, K. H. Lee, S. D. Pollard, Y. J. Shin, H. Zhou, W. Peng, D. Lee, W. Meng, H. Yang, J. H. Han, M. Kim, Q. Lu, and T. W. Noh, Ferroelectrically Tunable Magnetic Skyrmions in Ultrathin Oxide Heterostructures, Nat. Mater. 17, 1087 (2018).

[9] A. N. Bogdanov and A. A. Shestakov, Inhomogeneous TwoDimensional Structures in Liquid Crystals, J. Exp. Theor. Phys. 86, 911 (1998).

[10] A. N. Bogdanov, U. K. Rößler, and A. A. Shestakov, Skyrmions in Nematic Liquid Crystals, Phys. Rev. E 67, 016602 (2003).

[11] A. O. Leonov, I. E. Dragunov, U. K. Rossler, and A. N. Bogdanov, Theory of Skyrmion States in Liquid Crystals, Phys. Rev. E 90, 042502 (2014).

[12] T. Moriya, Anisotropic Superexchange Interaction and Weak Ferromagnetism, Phys. Rev. 120, 91 (1960).

[13] I. Dzyaloshinsky, A Thermodynamic Theory of Weak Ferromagnetism of Antiferromagnetics, J. Phys. Chem. Solids 4, 241 (1958).

[14] Y. A. Bychkov and E. I. Rashba, Properties of a 2D Electron Gas with Lifted Spectral Degeneracy, JETP Lett. 39, 78 (1984).

[15] P. J. Ackerman, R. P. Trivedi, B. Senyuk, J. van de Lagemaat, and I. I. Smalyukh, Two-Dimensional Skyrmions and Other Solitonic Structures in Confinement-Frustrated Chiral Nematics, Phys. Rev. E 90, 012505 (2014).

[16] D. Foster, C. Kind, P. J. Ackerman, J.-S. B. Tai, M. R. Dennis, and I. I. Smalyukh, Two-Dimensional Skyrmion Bags in Liquid Crystals and Ferromagnets, Nat. Phys. 15, 655 (2019).

[17] H. R. O. Sohn, C. D. Liu, and I. Smalyukh, Schools of Skyrmions with Electrically Tunable Elastic Interactions, Nat. Commun. 10, 4744 (2019).

[18] D. C. Wright and N. D. Mermin, Crystalline Liquids: The Blue Phases, Rev. Mod. Phys. 61, 385 (1989).

[19] A. Fert, N. Reyren, and V. Cros, Magnetic Skyrmions: Advances in Physics and Potential Applications, Nat. Rev. Mater. 2, 17031 (2017).

[20] A. Duzgun, J. V. Selinger, and A. Saxena, Comparing Skyrmions and Merons in Chiral Liquid Crystals and Magnets, Phys. Rev. E 97, 062706 (2018).

[21] S.-Z. Lin, A. Saxena, and C. D. Batista, Skyrmion Fractionalization and Merons in Chiral Magnets with EasyPlane Anisotropy, Phys. Rev. B 91, 224407 (2015).

[22] X. Z. Yu, W. Koshibae, Y. Tokunaga, K. Shibata, Y. Taguchi, N. Nagaosa, and Y. Tokura, Transformation 
between Meron and Skyrmion Topological Spin Textures in a Chiral Magnet, Nature (London) 564, 95 (2018).

[23] A. Nych, J. Fukuda, U. Ognysta, S. Žumer, and I. Muevi, Spontaneous Formation and Dynamics of Half-Skyrmions in a Chiral Liquid-Crystal Film, Nat. Phys. 13, 1215 (2017).

[24] A. Saupe, On Molecular Structure and Physical Properties of Thermotropic Liquid Crystals, Mol. Cryst. 7, 59 (1969).

[25] Liquid Crystals of One and Two-Dimensional Order, edited by W. Helfrich and G. Heppke (Springer, Heidelberg, 1980).

[26] M. Marcus, Quasicrystalline Behaviour and Phase Transition in Cholesteric Blue Phase, J. Phys. 42, 61 (1981).

[27] P. J. Collings, Optical Rotatory Dispersion Measurements in the Third Cholesteric Blue Phase, Phys. Rev. A 30, 1990 (1984).

[28] Z. Kutnjak, C. W. Garland, J. L. Passmore, and P. J. Collings, Supercritical Conversion of the Third Blue Phase to the Isotropic Phase in a Highly Chiral Liquid Crystal, Phys. Rev. Lett. 74, 4859 (1995).

[29] J. Thoen, Adiabatic Scanning Calorimetric Results for the Blue Phases of Cholesteryl Nonanoate, Phys. Rev. A 37, 1754 (1988).

[30] Z. Kutnjak, C. W. Garland, C. G. Schatz, P. J. Collings, C. J. Booth, and J. W. Goodby, Critical Point for the Blue Phase III-Isotropic Phase Transition in Chiral Liquid Crystals, Phys. Rev. E 53, 4955 (1996).

[31] E. P. Koistinen and P. H. Keyes, Light-Scattering Study of the Structure of Blue Phase III, Phys. Rev. Lett. 74, 4460 (1995).

[32] U. Singh, P. J. Collings, C. J. Booth, and J. W. Goodby, Static and Dynamic Light Scattering near the Liquid Crystalline Blue Phase III-Isotropic Liquid Critical Point, J. Phys. II (France) 7, 1683 (1997).

[33] P. J. Collings, Optical Activity and Light Scattering in Highly Chiral Liquid Crystals, Mod. Phys. Lett. B 06, 425 (1992).

[34] T. K. Brog and P. J. Collings, Optical Activity in the Blue Phase of a Cholesteric Liquid Crystal, Mol. Cryst. Liq. Cryst. 60, 65 (1980).

[35] J. B. Becker and P. J. Collings, Optical Measurements on the BP III to Isotropic Phase Transition in Highly Chiral Liquid Crystals, Mol. Cryst. Liq. Cryst. 265, 163 (1995).

[36] D. K. Yang and P. P. Crooker, Chiral-Racemic Phase Diagrams of Blue-Phase Liquid Crystals, Phys. Rev. A 35, 4419 (1987).

[37] T. C. Lubensky and H. Stark, Theory of a Critical Point in the Blue-Phase-III-Isotropic Phase Diagram, Phys. Rev. E 53, 714 (1996).

[38] M. A. Anisimov, V. A. Agayan, and P. J. Collings, Nature of the Blue-Phase-III-Isotropic Critical Point: An Analogy with the Liquid-Gas Transition, Phys. Rev. E 57, 582 (1998).

[39] R. M. Hornreich and S. Shtrikman, Broken Icosahedral Symmetry: A Quasicrystalline Structure for Cholesteric Blue Phase III, Phys. Rev. Lett. 57, 1963(E) (1986).

[40] L. Longa, W. Fink, and H. R. Trebin, Liquid-Crystalline Blue Phase III and Structures of Broken Icosahedral Symmetry, Phys. Rev. E 48, 2296 (1993).

[41] L. Longa and H. R. Trebin, Bond Orientational Order in the Blue Phases of Chiral Liquid Crystals, Phys. Rev. Lett. 71, 2757 (1993).
[42] D. S. Rokhsar and J. P. Sethna, Quasicrystalline Textures of Cholesteric Liquid Crystals: Blue Phase III?, Phys. Rev. Lett. 56, 1727 (1986).

[43] O. Henrich, K. Stratford, M. E. Cates, and D. Marenduzzo, Structure of Blue Phase III of Cholesteric Liquid Crystals, Phys. Rev. Lett. 106, 107801 (2011).

[44] O. Henrich, K. Stratford, D. Marenduzzo, and M. E. Cates, Ordering Dynamics of Blue Phases Entails Kinetic Stabilization of Amorphous Networks, Proc. Natl. Acad. Sci. U.S.A. 107, 13212 (2010).

[45] J. A. N. Zasadzinski, S. Meiboom, M. J. Sammon, and D. W. Berreman, Freeze-Fracture Electron-Microscope Observations of the Blue Phase III, Phys. Rev. Lett. 57, 364 (1986).

[46] F. Livolant and A. Leforestier, Condensed Phases of DNA: Structures and Phase Transitions, Prog. Polym. Sci. 21, 1115 (1996).

[47] S. S. Gandhi, M. S. Kim, J.-Y. Hwang, and L.-C. Chien, Electro-Optical Memory of a Nanoengineered Amorphous Blue Phase III Polymer Scaffold, Adv. Mater. 28, 8998 (2016).

[48] See Supplemental Material at http://link.aps.org/ supplemental/10.1103/PhysRevX.12.011003 for experimental videos of observed LC skyrmion structures.

[49] J. Englert, L. Longa, H. Stark, and H. R. Trebin, Fluctuations Dominate the Phase Diagram of Chiral Nematic Liquid Crystals, Phys. Rev. Lett. 81, 1457 (1998).

[50] T. Harada and P. P. Crooker, Light Scattering through the Isotropic-Cholesteric Phase Transition of a Cholesteric Liquid Crystal, Phys. Rev. Lett. 34, 1540 (1975).

[51] D. S. Mahler, P. H. Keyes, and W. B. Daniels, Light-Scattering Study of the Pretransitional Phenomena in the Isotropic Phase of Cholesteryl Oleyl Carbonate (COC), Phys. Rev. Lett. 36, 491 (1976).

[52] P. H. Keyes and C. C. Yang, Dynamics of the IsotropicCholesteric Transition: Differences between the Normal and Blue Cholesteric Phases, J. Phys. (Paris), Colloq. 40, C3 (1979).

[53] M. Kleman and J. Friedel, Disclinations, Dislocations, and Continuous Defects: A Reappraisal, Rev. Mod. Phys. 80, 61 (2008).

[54] S. P. Thampi, R. Golestanian, and J. M. Yeomans, Vorticity, Defects and Correlations in Active Turbulence, Phil. Trans. R. Soc. A 372, 20130366 (2014).

[55] A. Doostmohammadi, J. Ignés-Mullol, J. M. Yeomans, and F. Sagués, Active Nematics, Nat. Commun. 9, 3246 (2018).

[56] S. Čopar, J. Aplinc, Ž. Kos, S. Žumer, and M. Ravnik, Topology of Three-Dimensional Active Nematic Turbulence Confined to Droplets, Phys. Rev. X 9, 031051 (2019).

[57] G. Duclos, R. Adkins, D. Banerjee, M. S. E. Peterson, M. Varghese, I. Kolvin, A. Baskaran, R. A. Pelcovits, T. R. Powers, A. Baskaran, F. Toschi, M. F. Hagan, S. J. Streichan, V. Vitelli, D. A. Beller, and Z. Dogic, Topological Structure and Dynamics of Three-Dimensional Active Nematics, Science 367, 1120 (2020).

[58] T. Sanchez, D. T. N. Chen, S. J. DeCamp, M. Heymann, and Z. Dogic, Spontaneous Motion in Hierarchically Assembled Active Matter, Nature (London) 491, 431 (2012). 
[59] L. N. Carenza, G. Gonnella, D. Marenduzzo, and G. Negro, Rotation and Propulsion in 3D Active Chiral Droplets, Proc. Natl. Acad. Sci. U.S.A. 116, 22065 (2019).

[60] J. Pišljar, G. Posnjak, S. Pajk, A. Godec, R. Podlipec, B. Kokot, and I. Muševič, Comparison of STED, Confocal and Optical Microscopy of Ultra-short Pitch Cholesterics, Liq. Cryst. 47, 1303 (2020).

[61] S. Tewari, D. Belitz, and T. R. Kirkpatrick, Blue Quantum Fog: Chiral Condensation in Quantum Helimagnets, Phys. Rev. Lett. 96, 047207 (2006).

[62] C. Pappas, E. Lelièvre-Berna, P. Falus, P. M. Bentley, E. Moskvin, S. Grigoriev, P. Fouquet, and B. Farago, Chiral Paramagnetic Skyrmion-like Phase in MnSi, Phys. Rev. Lett. 102, 197202 (2009).

[63] X. Zhang, Y. Zhou, K. M. Song, T.-E. Park, J. Xia, M. Ezawa, X. Liu, W. Zhao, G. Zhao, and S. Woo, SkyrmionElectronics: Writing, Deleting, Reading and Processing Magnetic Skyrmions toward Spintronic Applications, J. Phys. Condens. Matter 32, 143001 (2020).

[64] P. J. Ackerman, T. Boyle, and I. I. Smalyukh, Squirming Motion of Baby Skyrmions in Nematic Fluids, Nat. Commun. 8, 673 (2017).

[65] I. Smalyukh, S. Shiyanovskii, and O. Lavrentovich, ThreeDimensional Imaging of Orientational Order by Fluorescence Confocal Polarizing Microscopy, Chem. Phys. Lett. 336, 88 (2001).

[66] M. Škarabot, Ž. Lokar, K. Gabrijelčič, D. Wilkes, and I. Muševič, Atomic Force Microscope Based Method of Measuring Short Cholesteric Pitch in Liquid Crystals, Liq. Cryst. 38, 1017 (2011).

[67] R. K. Khan, S. Turlapati, N. V. S. Rao, R. Pratibha, W. Drzewinski, R. Dabrowski, and S. Ghosh, Novel Achiral Four-Ring Bent-Shaped Nematic Liquid Crystals with Trifluoromethyl and Methyl Substituents in the Central Molecular Core: An Unusually Large Kerr Constant in Blue Phase III of Nematic-Chiral Dopant Mixture, J. Mater. Chem. C 5, 6729 (2017).

[68] M. Škarabot, M. Čepič, B. Žekš, R. Blinc, G. Heppke, A. V. Kityk, and I. Muševič, Birefringence and Tilt Angle in the Antiferroelectric, Ferroelectric, and Intermediate Phases of Chiral Smectic Liquid Crystals, Phys. Rev. E 58, 575 (1998).

[69] P. B. J. Berne and R. Pecora, Dynamic Light Scattering: With Applications to Chemistry, Biology, and Physics (Dover, New York, 2000).

[70] P. D. Kaplan, V. Trappe, and D. A. Weitz, Light-Scattering Microscope, Appl. Opt. 38, 4151 (1999).
[71] M. T. Valentine, A. K. Popp, D. A. Weitz, and P. D. Kaplan, Microscope-Based Static Light-Scattering Instrument, Opt. Lett. 26, 890 (2001).

[72] M. S. Amin, Y. Park, N. Lue, R. R. Dasari, K. Badizadegan, M. S. Feld, and G. Popescu, Microrheology of Red Blood Cell Membranes Using Dynamic Scattering Microscopy, Opt. Express 15, 17001 (2007).

[73] W. J. Cottrell, J. D. Wilson, and T. H. Foster, Microscope Enabling Multimodality Imaging, Angle Resolved Scattering, and Scattering Spectroscopy, Opt. Lett. 32, 2348 (2007).

[74] R. Cerbino and V. Trappe, Differential Dynamic Microscopy: Probing Wave Vector Dependent Dynamics with a Microscope, Phys. Rev. Lett. 100, 188102 (2008).

[75] J. Baumgartl, J. Dietrich, J. Dobnikar, C. Bechingerac, and H. H. von Gruenberg, Phonon Dispersion Curves of Two-Dimensional Colloidal Crystals: The WavelengthDependence of Friction, Soft Matter 4, 2199 (2008).

[76] M. Arko and A. Petelin, Cross-Differential Dynamic Microscopy, Soft Matter 15, 2791 (2019).

[77] A. Petelin, Cross-Differential Dynamic Microscopy, v. $0.1 .2,2020$.

[78] C. P. Lindsey and G. D. Patterson, Detailed Comparison of the Williamswatts and Coledavidson Functions, J. Chem. Phys. 73, 3348 (1980).

[79] P. G. de Gennes and J. Prost, Physics of Liquid Crystals (Oxford University, New York, 1995).

[80] M. Ravnik, G. P. Alexander, J. M. Yeomans, and S. Zumer, Mesoscopic Modelling of Colloids in Chiral Nematics, Faraday Discuss. 144, 159 (2010).

[81] J. B. Fournier and P. Galatola, Modeling Planar Degenerate Wetting and Anchoring in Nematic Liquid Crystals, Europhys. Lett. 72, 403 (2005).

[82] A. Dupuis, D. Marenduzzo, and J. M. Yeomans, Numerical Calculations of the Phase Diagram of Cubic Blue Phases in Cholesteric Liquid Crystals, Phys. Rev. E 71, 011703 (2005).

[83] W. J. A. Goossens, Order Electricity Reconsidered, Liq. Cryst. 5, 1083 (1989).

[84] M. D. Feit and J. A. Fleck, Light Propagation in GradedIndex Optical Fibers, Appl. Opt. 17, 3990 (1978).

[85] A. F. Oskooi, D. Roundy, M. Ibanescu, P. Bermel, J. D. Joannopoulos, and S. G. Johnson, Meep: A Flexible FreeSoftware Package for Electromagnetic Simulations by the FDTD Method, Comput. Phys. Commun. 181, 687 (2010).

[86] A. Bregar, T. J. White, and M. Ravnik, Refraction of Light on Flat Boundary of Liquid Crystals or Anisotropic Metamaterials, Liq. Cryst. Rev. 5, 53 (2017). 